\title{
Glass Transition in Supercooled Liquids with Medium Range Crystalline Order
}

\author{
Indrajit Tah ${ }^{1}$, Shiladitya Sengupta ${ }^{2}$, Srikanth Sastry ${ }^{3}$, Chandan Dasgupta ${ }^{4}$, and Smarajit Karmakar ${ }^{1}$ \\ 1 Centre for Interdisciplinary Sciences, Tata Institute of Fundamental Research, \\ 21 Brundavan Colony, Narisingi, Hyderabad, 500075, India, \\ 2 Department of Chemical Physics, Weizmann Institute of Science, Israel, \\ 3 Jawaharlal Nehru Centre for Advanced Scientific Research, Bangalore 560064, India, \\ 4 Centre for Condensed Matter Theory, Department of Physics, \\ Indian Institute of Science, Bangalore, 560012, India
}

\begin{abstract}
The origins of rapid dynamical slow down in glass forming liquids in the growth of static length scales, possibly associated with identifiable structural ordering, is a much debated issue. Growth of medium range crystalline order (MRCO) has been observed in various model systems to be associated with glassy behaviour. Such observations raise the question about the eventual state reached by a glass former, if allowed to relax for sufficiently long times. Is a slowly growing crystalline order responsible for slow dynamics? Are the molecular mechanisms for glass transition in liquids with and without MRCO the same? If yes, glass formers with MRCO provide a paradigm for understanding glassy behaviour generically. If not, systems with MRCO form a new class of glass forming materials whose molecular mechanism for slow dynamics may be easier to understand in terms of growing crystalline order, and should be approached in that manner, even while they will not provide generic insights. In this study we perform extensive molecular dynamics simulations of a number of glass forming liquids in two dimensions and show that the static and dynamic properties of glasses with MRCO are different from other glass forming liquids with no predominant local order. We also resolve an important issue regarding the so-called Point-to-set method for determining static length scales, and demonstrate it to be a robust, order agnostic, method for determining static correlation lengths in glass formers.
\end{abstract}

The existence of growing length scales and their connection to the rapid slowing down of dynamics of glass forming liquids approaching glass transition are still a subject of active research. Based on extensive research on the existence of various static and dynamical length scales in glass formers, it is now understood that multiple growing length scales exist and correlate with increasing relaxation time scales as the glass transition is approached, but whether a single or a small number of such length scales satisfactorily describe the essential physics of dynamical slow down and the glass transition is not fully resolved [1-6]. For quite sometime in the study of glass formers, there has been a focus on identifying structural motifs which are favoured locally but which can not span the whole system due to frustration arising from the incommensurate nature of the structural motifs. There are many experimental and simulation studies which claim the existence of such structural motifs in a variety of glass forming liquids $[7,8]$. A strong connection between the growth of such locally favoured structures (also widely known as medium range crystalline order, MRCO) and slow relaxation has also been proposed in some glass formers, with a fair degree of supporting evidence [9-11]. In Figure 1, snapshot of such MRCO (red regions in the figure) is shown for one of the model glass formers studied in this work. While the dynamics of different glass forming liquids are very similar to each other in essential aspects, only some glass formers manifest growing MRCO, whereas such structural motifs can not be clearly identified in many others. The failure to identify growing structural order may either be due to their absence or due to the subtlety of the relevant structural order that may not be captured by the metrics employed. It is thus important to understand whether the physics that govern the slowing down of dynamics in different glass forming liquids are generically the same irrespective of the presence of well characterised MRCO or whether essential differences can be found between those systems with MRCO and those without. If it turns out that indeed the physics governing the slowing down in different glass forming liquids are same and all glass forming liquids eventually will start to have locally preferred structurally ordered domains, then the complexity of glass transition will be reduced to the formation of such domains and efforts must be directed at detecting through suitable metrics the growing structural order. On the other hand if it turns out that the behaviour of glass formers with MRCO is not generic, then they form a distinguishable special class of glass forming systems, whose behaviour is perhaps less complex, and must be analysed differently.

Recently, in a series of papers Tanaka et. al. [9-11] showed that the glass transition in systems with MRCO is determined by the length scale associated with that of locally preferred structures. For example, for two dimensional systems, the correlation length associated with hexatic order is the important length scale and the glass transition in these systems are completely controlled by the hexatic correlation length. In [12], a polydisperse two dimensional glass forming liquid was studied in which the hexatic correlation grows rapidly with increasing packing fraction. The dynamic heterogeneity [13-15] length scale obtained from the scaling analysis of the wave-vector $(q)$ dependence of the four-point structure factor, $S_{4}(q, t)$ 
[16] was compared with the hexatic correlation length obtained from the spatial correlation function of the hexatic order parameter, $\psi_{6}[11,17,18]$ defined as

$$
\psi_{6}=\frac{1}{N} \sum_{i=1}^{N} \psi_{6}^{i}, \quad \text { where } \quad \psi_{6}^{j}=\frac{1}{n_{j}} \sum_{k=1}^{n_{j}} \exp \left(\imath 6 \theta_{j k}\right)
$$

where $\theta_{j k}$ is the angle made by the vector from particle $j$ to its neughbour $k$ with the $x$-axis and $n_{j}$ is the number of neighbours of particle $j$ (see SI for further details). These two length scales were found to be proportional to each other over the studied density range.

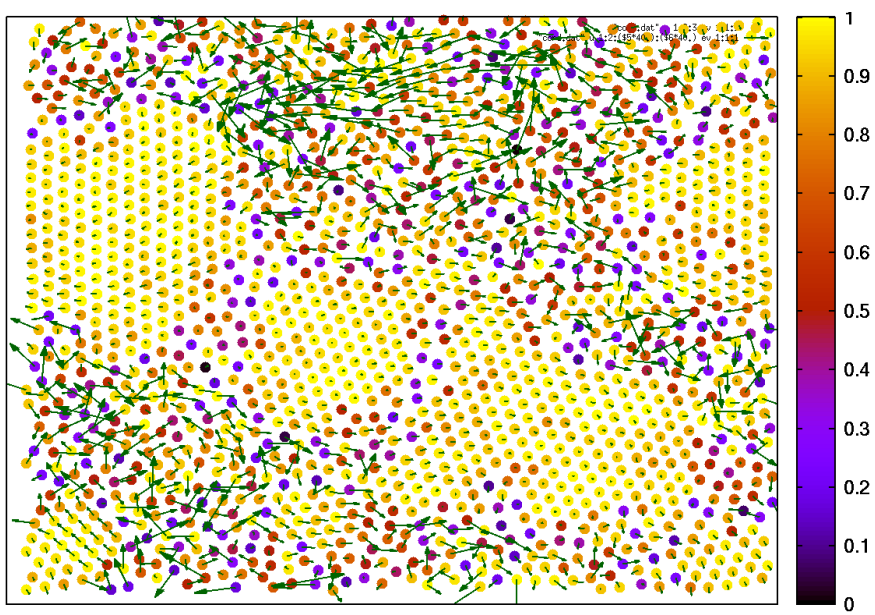

FIG. 1. Dynamic heterogeneity and MRCO for 2dKA syatem. A snapshot of the system with $N=2000$ at $T=$ 0.930 and $\rho=1.20$ with particles coloured according to their local hexatic order parameter $\left|\psi_{6}^{i}\right|$, as indicated in the right side colour bar of the figure. The arrows are the displacements of particles calculated over $\alpha$ relaxation time starting from this configuration.

The static length scale obtained from few different methods including the Point-to-set (PTS) [19, 20] method was also compared with the hexatic correlation length. The PTS length scale was found to be very different from the hexatic correlation length in the studied density window. In this system with local hexatic order, the behaviour is completely determined by the hexatic correlation length. So, if the PTS method is "order agnostic", then it should pick up the same hexatic correlation length. From the large difference between the calculated values of the PTS and hexatic length scales, it was concluded that the PTS method fails to pick up the relevant static length scale for systems with MRCO. These results cast a serious doubt about the usefulness of the PTS method in measuring important static length scales relevant for slow dynamics in all glass forming liquids.

In this work we have addressed the important issue of whether a deep connection between structure and dynamics exists for all glass forming liquids. As noted above, a number of theoretical approaches [3, 21-24] have explored the relevance of a growing length scale to dynamical slow down. A specific goal in some of these approaches were to study the connection between heterogeneous dynamics (also referred to as dynamic heterogeneity) in glass formers [4, 25-27] with rapid change in viscosity. We show that the slowing down in the dynamics while approaching the glass transition in liquids which exhibit a pronounced increase in structural order is strongly coupled to the growth of dynamic heterogeneity length scales, as well as to the growth of the length scale that captures the local structural order. In twodimensional glass formers with MRCO, the static length scale determined by growing hexatic order is the same as both the dynamic heterogeneity length scale and a static amorphous length scale which we independently evaluate using the PTS method. On the other hand, for liquids in which such structural ordering is not prominent, the temperature dependence of the dynamic heterogeneity length scale is very different from that of the static amorphous length scale. This suggests that the glass transition in liquids which show a strong tendency to the growth of locally favoured structural order is fundamentally different from the transition in liquids in which such local crystalline structures do not grow very prominently.

We address these issues by performing extensive computer simulations of four different glass forming liquids in two dimensions and measuring all the relevant length scales in each system using a wide range of procedures. The model systems studied are: (i) the two-dimensional Kob-Andersen binary Lennard-Jones mixture (referred here as 2dKA) [28] at fixed density, with the temperature as the variable that determines changes in dynamics; (ii) a two- dimensional system characterized by a repulsive inverse power-law potential, referred to as 2dIPL [29], studied with density as the relevant variable. For the above two systems, structural order or MRCO grows significantly while approaching the putative glass transition. We study another system (iii) with a repulsive interaction potential (referred to as 2dR10) [30]. No pronounced crystalline order is found in the studied temperature range for this model. This is consistent with previous work [31] that showed that the 2dR10 model has almost no tendency to exhibit locally favored crystalline order. We also study (iv) the polydisperse system studied by Tanaka et al [12], for reasons which we discuss later. The details of the models and simulations are given in the SI.

Rest of the paper is organized as follows: first, we briefly discuss the systems with local hexatic order (2dKA, 2dIPL) and compare various length scales in those model systems. We then discuss the model (2dR10) for which there is no prominent hexatic order and compare the findings with those for the previous set of model systems. Then we will discuss the controversy regarding the PTS method and describe results that resolve that controversy. Finally, we conclude with a summary of the perspective regarding the relationship between structural order and dynamics in different classes of glass formers. 

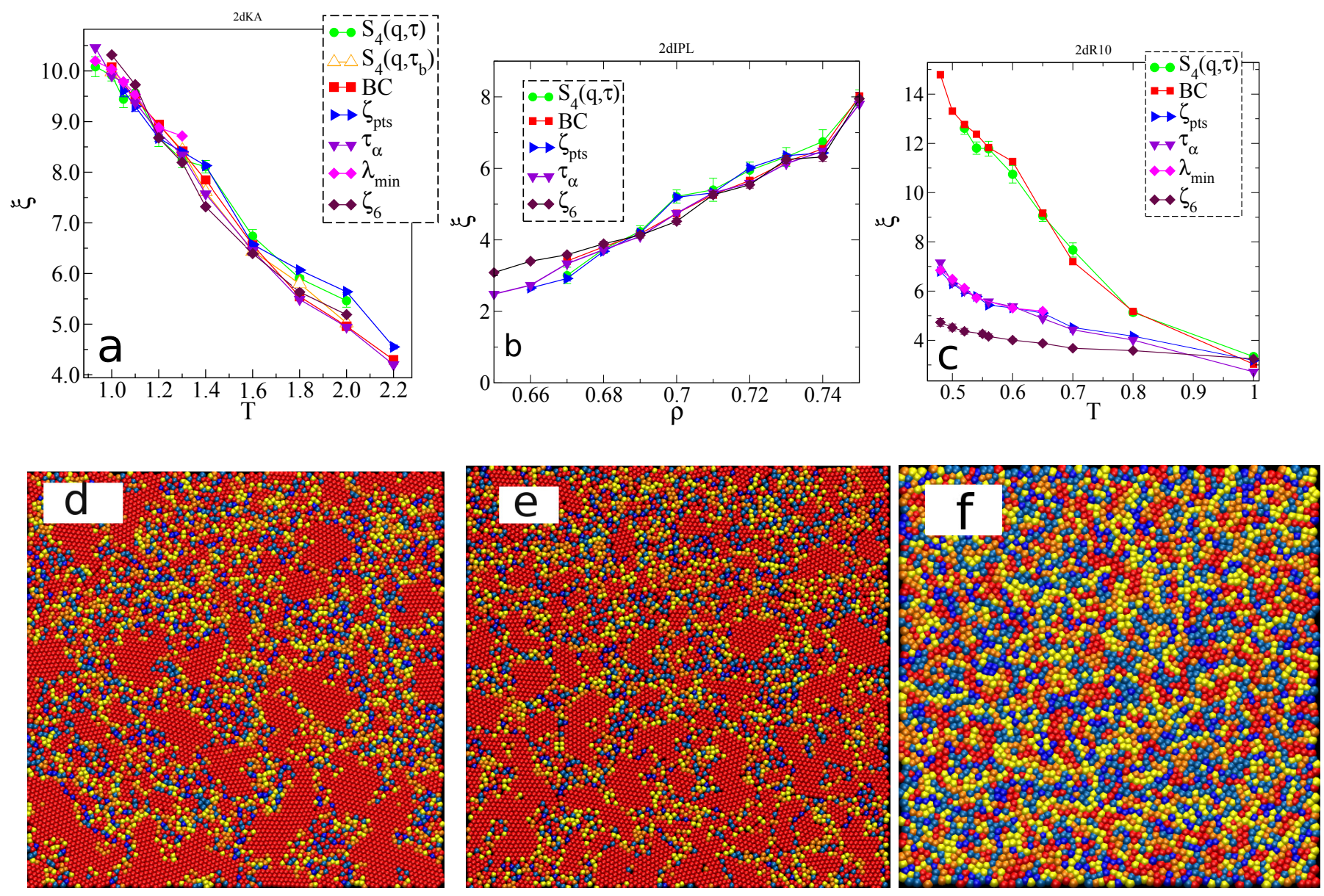

FIG. 2. Growth of dynamical and static length scales for three different systems 2dKA(a), 2dIPL(b), and 2dR10 (c). Snapshots show hexatic order for the same systems (d, e, f). (a) Correlation lengths as a function of temperature $T$ for the $2 \mathrm{dKA}$ system. Symbols for different quantities are as follows: Green circle for the dynamical length scale $\xi_{d}$ are obtained from $S_{4}(q, \tau)$; Orange triangles for the dynamical length scale obtained from $S_{4}\left(q, \tau_{b}\right)$ (details in SI); Red squares for dynamical length scale $\xi_{d}$ calculated by Binder cumulant scaling; Blue right triangle for PTS length scale $\xi_{p t s}$; Purple lower triangle for static length scales obtained from finite size scaling of $\tau_{\alpha}$; Pink diamonds for the static length scale obtained from the smallest eigenvalue of the Hessian matrix; Brown diamond for hexatic correlation length $\xi_{6}$. Same colours and symbols are used in the other two panels. (b) Correlation lengths as a function of density $\rho$ for the 2 dIPL system. (c) Correlation lengths as a function of temperature $T$ for the $2 \mathrm{dR} 10$ system. (d) Snapshot of a configuration at $\mathrm{T}=1.0$ (2dKA) in which the particles are coloured according to the following criteria: Red for very high hexatic order $\left(\left(\left|\psi_{6}^{i}\right|\right) \geq 0.80\right)$; Orange for high hexatic order $\left(\left(\left|\psi_{6}^{i}\right|\right) \geq 0.60\right.$ and $\left.\left(\left|\psi_{6}^{i}\right|\right)<0.80\right)$; Yellow for medium hexatic order $\left(\left(\left|\psi_{6}^{i}\right|\right) \geq 0.40\right.$ and $\left.\left(\left|\psi_{6}^{i}\right|\right)<0.60\right)$; Light blue for low hexatic order $\left(\left(\left|\psi_{6}^{i}\right|\right)\right.$ $\geq 0.20$ and $\left.\left(\left|\psi_{6}^{i}\right|\right)<0.40\right)$; Dark blue for very low hexatic order $\left(\left(\left|\psi_{6}^{i}\right|\right) \geq 0.0\right.$ and $\left.\left(\left|\psi_{6}^{i}\right|\right)<0.20\right)$. Same colouring is used in the following two panels. (e) Snapshot of a configuration at $\rho=0.74$ (2dIPL), (f) Snapshot of a configuration at $T=0.500$ (2dR10).

For the 2dKA model, hexatic order grows with decreasing temperature as illustrated in Figure 1 where particles are coloured according to the value of the local hexatic order parameter, $\psi_{6}^{i}$. Similarly, hexatic order grows for the 2dIPL model with increasing density. We have extracted the correlation length associated with the hexatic order by fitting the decay profile of the peaks of the hexatic correlation function, $g_{6}(r)$ [32] defined as

$$
g_{6}(r)=\left\langle\psi_{6}^{*}(\vec{r}) \psi_{6}(0)\right\rangle,
$$

where $\psi_{6}(\vec{r})=\sum_{i=1}^{N} \delta\left(\vec{r}-\vec{r}_{i}\right) \psi_{6}^{i}$. We have normalized it by pair correlation function $g(r)$ (see SI for further details). We have used exponential function and the two dimensional version of the Ornstein-Zernike function, to fit the decay of the peaks of the correlation function to get the correlation length. The correlation length $\xi_{6}$ is plotted in Figure 2 (a), 2 (b) and 2 (c) together with other dynamical and static correlation lengths which are described below.

Previous studies have shown $[10,11]$ a one-to-one correspondence between dynamic heterogeneities and regions of high/low hexatic order. We also find the same correspondence for the model systems studied here where medium range hexatic correlations are prominent. We 
have calculated the dynamical length scale $\xi_{4}$ from the four point structure factor $S_{4}(q, t)[16,22]$, which is defined as

$$
S_{4}(q, t)=N\left[\langle\tilde{Q}(q, t) \tilde{Q}(-q, t)\rangle-\langle\tilde{Q}(q, t)\rangle^{2}\right]
$$

with $\tilde{Q}(q, t)$ is defined as

$$
\tilde{Q}(q, t)=\frac{1}{N} \sum_{i=1}^{N} \exp \left(i \mathbf{q} \cdot \mathbf{r}_{i}(0)\right) w\left(\left|\vec{r}_{i}(t)-\vec{r}_{i}(0)\right|\right)
$$

where the window function, $w(x)=1$ if $x<0.3$ and 0 otherwise.

A dynamical susceptibility $\chi_{4}(t)$ is defined in terms of the fluctuations of two point overlap correlation function $Q(t)$ as

$$
\chi_{4}(t)=N\left[\left\langle Q^{2}(t)\right\rangle-\langle Q(t)\rangle^{2}\right] .
$$

The function $Q(t)$ (see SI for further details) defined as

$$
Q(t)=\frac{1}{N} \sum_{i=1}^{N} w\left(\left|\vec{r}_{i}(t)-\vec{r}_{i}(0)\right|\right),
$$

This function measures the overlap of a configuration of particles at a given initial time $(t=0)$ with the configuration at a later time $t$. Moreover $\chi_{4}(t) \equiv \lim _{q \rightarrow 0} S_{4}(q, t)$. The $\alpha$ relaxation time $\tau_{\alpha}$ is defined as $Q\left(t=\tau_{\alpha}\right)=1 / e$. We define another time scale $\tau$ as the time at which $\chi_{4}(t)$ peaks - it is proportional to $\tau_{\alpha}$ with a proportionality constant close to 1 . We consider $S_{4}(q, t)$ evaluated as $t=\tau$ in our analysis using the Ornstein-Zernike form,

$$
S_{4}(q, \tau)=\chi_{0} \mathcal{F}\left(q \xi_{4}\right),
$$

to obtain the dynamical correlation length, $\xi_{4}$. Here, $\chi_{0}=S_{4}(q=0, \tau)$ is the $q=0$ value of the structure factor and is same as $\chi_{4}(\tau)$ only in the Grand canonical Ensemble [3, 33-35], and $\mathcal{F}(x)$ is a scaling function (see SI for further details of the scaling analysis).

We have also estimated the dynamical correlation length $\xi_{4}$ from detailed finite size scaling analysis of the Binder Cumulant [3, 11, 36, 37], defined as

$$
B(N, T)=1-\frac{<Q(N, T)_{\tau}^{4}>}{3<Q(N, T)_{\tau}^{2}>^{2}},
$$

where $Q(N, T)_{\tau} \equiv Q(t=\tau)$ for system size $N$ at temperature $T$. The Binder cumulant is defined in terms of $4^{\text {th }}$ and $2^{\text {nd }}$ moments of the distribution of $Q$ so that it becomes zero for a Gaussian distribution and acquires positive values for bimodal distributions. It is a scaling function of only $L / \xi_{4}$ where $L$ is the linear dimension of the system (further details can be found in SI). From Figures 2.(a), 2.(b) and 2.(c) it is clear that this length scale obtained from finite size scaling of $B(N, T)$ is in good agreement with the dynamical length scale calculated from the four-point structure factor $S_{4}(q, \tau)$. For one model (2dKA) we also compute the dynamical length scale from the four-point structure factor of bond breakage correlation function (see SI for details), and as seen in Figure. 2 (a), this length scale also compares very well with the lengths obtained by the other methods.

The growth of the calculated dynamical correlation length, $\xi_{4}$ is found to be in good agreement with the hexatic correlation length, $\xi_{6}$ for models $2 \mathrm{dKA}$ and $2 \mathrm{dIPL}$. This is in complete agreement with the observation made in Ref.[12] where it was shown that for systems with growing hexatic order, the hexatic correlation length is the same as the dynamical heterogeneity length scale.

To understand possible connections between the growing hexatic order and static length scales associated with amorphous order, we have calculated the static length scale using three different methods: (a) PTS correlation method, (b) Finite size scaling of the relaxation time $\tau_{\alpha}$ and (c) Finite size scaling of the minimum eigenvalue of the Hessian matrix [31] calculated at local minima of the potential energy (known as Inherent structures) visited by the system at a given temperature. Computing PTS correlations $[19,20]$ is an elegant, general method to capture the multi-point static structural correlations in viscous liquids. The main idea is to freeze the majority of particles from an equilibrated configuration of the liquid, outside a specified volume, and to measure how the structure of the unfrozen particles in the inner volume are affected. It has been argued that in the geometry in which particles outside a spherical cavity are frozen, the PTS correlation should detect the typical domain size predicted by the random first order transition theory (RFOT) [38, 39]. Here we calculate the point to set length scale (referred to as $\xi_{P T S}$ ) in the cavity geometry. Bulk equilibrium configurations are generated at desired temperature and density using NVT Molecular dynamics simulations and then cavities are constructed by freezing the particles outside a circular region of radius $R$. Static overlap correlations are calculated as a function of cavity radius $R$ and from the decay profile we obtain the PTS length scale (see SI for further details).

The static length scale obtained from the finite size scaling of the relaxation time is referred to here as $\xi_{\tau}$ $[2,36]$. The details of the analysis and the scaling plots are given in the SI. The length scale $\xi_{\lambda}$ obtained from the scaling of the minimum eigenvalue of the Hessian matrix is calculated only at low temperatures due to the harmonic nature of the analysis which will not be a good approximation at higher temperatures (see the SI for further details of the analysis and scaling plots for different model systems).

The comparison of different length scales are summarized in Figures 2.(a), 2.(b) and 2.(c). The data for 2dKA and 2dIPL in which MRCO grows significantly, both dynamic and static length scales obtained using different methods grow very similarly as the hexatic correlation length. On the other hand for the 2dR10 model where no significant hexatic order is observed in the studied temperature range, the dynamic length scales grow 
much faster than both the static length scale and the hexatic length scale. Note that for the $2 \mathrm{dR} 10$ model, the growth of the hexatic correlation length is even smaller than the modest growth of the static length scale.

These results clearly show that the relationship between dynamical, static and hexatic order length scales is significantly different for glass formers with MRCO (for which all length scales are comparable) and those without (for which different length scales are significantly different).

We next consider the relationship between the dynamical length scales and relaxation times. In Figure. 3 we plot the relaxation time (scaled by its value in the infinite size limit) against the Binder cumulant for the three different models, and for different temperatures (or densities for the 2dIPL model) and system sizes in each case. If the system studied exhibits dynamical finite-size scaling, the data for different system sizes and temperatures should fall on a master curve. Further, since we expect the relaxation time to be governed by a static length scale (see above), such a data collapse would also indicate the equality of static and dynamical length scales, which is not generally expected to hold. Figure. 3 shows $\tau_{\alpha}(N, T) / \tau_{\alpha}(N \rightarrow \infty, T)$ vs. $B(N, T)$ for $2 \mathrm{dKA}$ and 2dR10 model and $\tau_{\alpha}(N, \rho) / \tau_{\alpha}(N \rightarrow \infty, \rho)$ vs. $B(N, \rho)$ for the 2dIPL model systems we study. The top two panels, for 2dKA and 2dIPL, show convincing data collapse, indicating that a unique length scale, which we may attribute to growing hexatic order, controls the dynamics, as well as the static order. In contrast, the bottom panel, for 2dR10, shows no data collapse, and confirms our previous observation in Figure. 3 that the growths of dynamic and static length scales are different and decoupled from each other in this system.

However, our conclusion about the equality of static, dynamic and structural length scales in systems exhibiting prominent hexatic order has recently been questioned. In Ref. [12], it was argued that the PTS method $[40,41]$ fails to capture the growth of the relevant static length scale for systems which exhibit growing MRCO. The system studied in Ref.[12] is a polydisperse one with $11 \%$ polydispersity. In the PTS method one calculates the static overlap as a function of different cavity radii (R) for a given temperature or density and then from the decay of the static overlap with increasing cavity radius one extracts the static length-scale.

For randomly chosen small cavities, the packing fraction and polydispersity will be distributed around the mean values, with the variance being larger for smaller cavities. It may be expected, however, that for a sufficiently large number of realisations, such that the average packing fraction and polydispersity lie within a reasonably small tolerance, the estimated properties (in this instance, the static correlations to obtain the PTS length scale) will converge to the correct values. We thus estimate the number of realisations required for the average polydispersity and packing fraction to lie within $2 \%$ of the bulk values, different values of the PTS length are
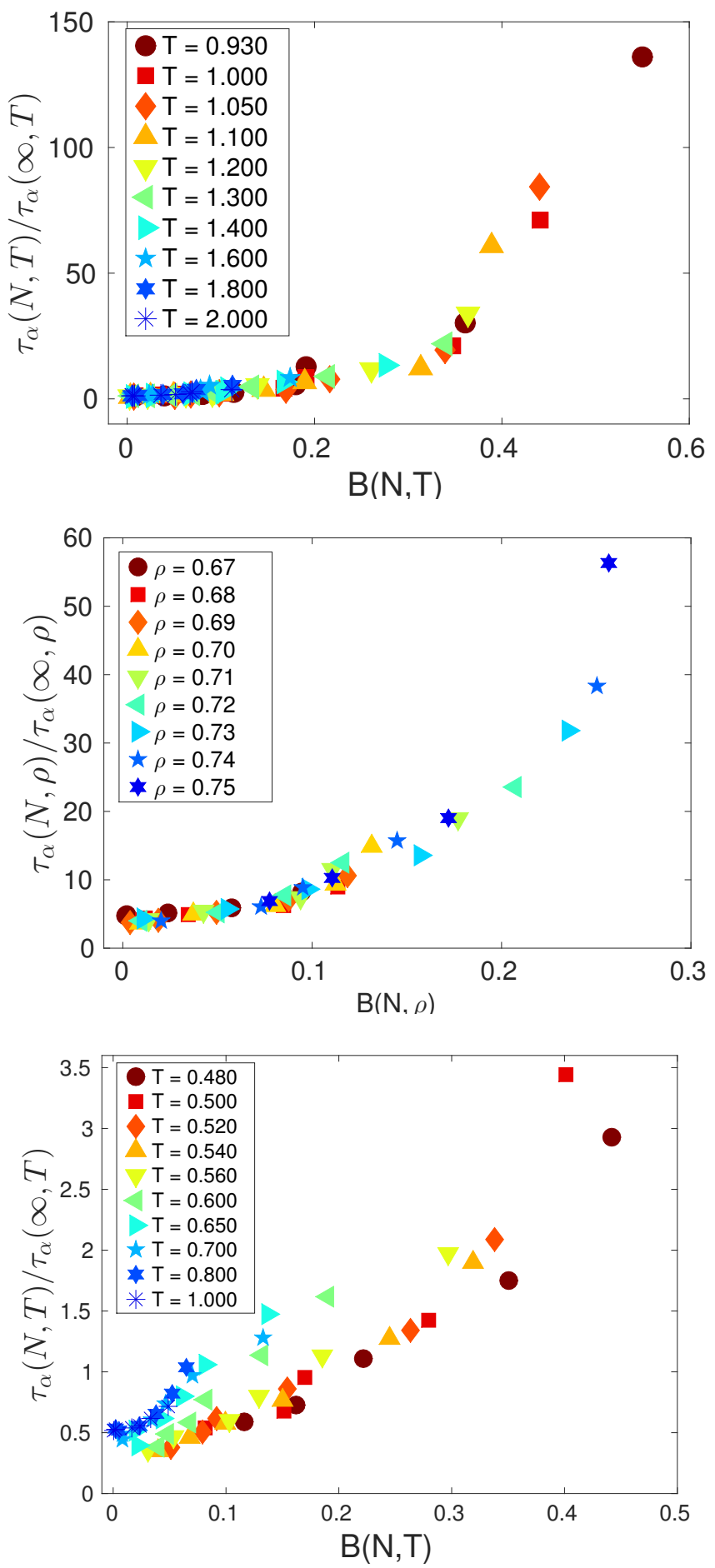

FIG. 3. The $\alpha$ relaxation time $\tau_{\alpha}(N, T)$ (or $\tau_{\alpha}(N, \rho)$ in the case of the 2dIPL model), scaled by its value for $N \rightarrow \infty$, plotted against the Binder cumulant $B(N, T)$ (or $B(N, \rho)$ in the case of the 2dIPL model), for 2dKA system (top) $2 \mathrm{dIPL}$ system (middle) and 2dR10 system (bottom).

obtained. In the inset of the left panel of Figure. 4 we show such an estimate for a system of $N=10000$ polydisperse particles in two dimension with $11 \%$ polydispersity (henceforth we will refer to this model as 2dPoly). One 

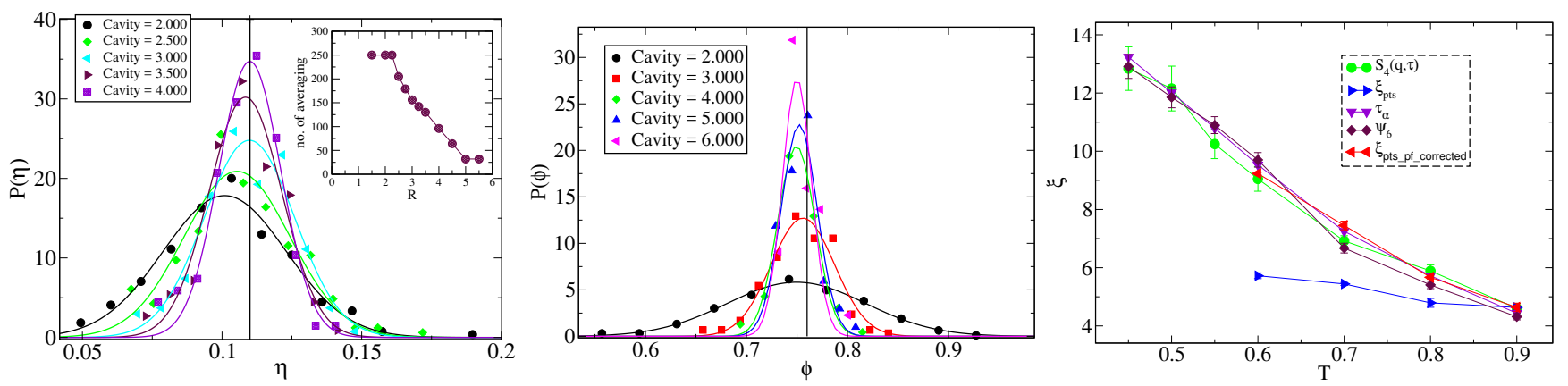

FIG. 4. Left Panel: Probability distribution of polydispersity for cavities of different radii. The black vertical line denotes the mean polydispersity of $11 \%$. Inset shows the number of samples required to get the average polydispersity within $2 \%$ of the bulk value. Middle Panel: Probability distribution of packing fraction. The black vertical line denotes the bulk packing fraction 0.76. Labels for different data sets are the radii of the cavities. Right Panel: Comparison of different length scales for the 2dPoly system showing that the corrected PTS length agrees with other static and dynamical length scales computed.

can clearly see that for small cavities, one needs averaging over as large as 250 different cavities in order to reach the bulk polydispersity. The distributions of the polydispersity and packing fraction obtained for different cavity sizes are shown in the left and middle panels of Figure. 4.

We then obtained the PTS length scale by averaging over the estimated number of cavities for each radius. The results obtained this way are shown in the right panel of Figure.4 (blue triangles). Similarly to the results in Ref.[12], we find that the PTS length scale so estimated falls well below the hexatic length scale and other static and dynamical length scales that track the hexatic length scale. This result is not expected to change if we average over a larger number of cavities for each $R$.

If, on the other hand, we adopt, following [19, 20], the procedure of evaluating the PTS length scale by selecting only those cavities for any radius for which the packing fraction and polydispersity within the cavity fall within a cutoff of $2 \%$ from the bulk values. The PTS length scales obtained from this procedure (magenta triangle) match remarkably well the hexatic order length, the dynamical heterogeneity length scale and the static length scale estimated from finite size scaling of the relaxation time. We believe this procedure to be more appropriate because thermodynamic parameters such as temperature, density and polydispersity of the particles inside the cavity are assumed to be fixed in the theoretical arguments $[19,20]$ that relate the PTS length scale to the mosaic scale of RFOT. We thus conclude that the difference between the PTS length scale and the hexatic order length scale observed in Ref. [12] arises from the method of calculation, rather than from any fundamental limitations of the PTS method in determining static length scales. The PTS method appears to be a robust, order agnostic approach, and while the issues surrounding what constitutes a proper way of calculating $\xi_{P T S}$ need to be better understood, there may be no need to modify it, as recently proposed [42].

In conclusion, we have shown that the growth of different measures of static and dynamic length scales in supercooled liquids with medium range crystalline order (MRCO) is fundamentally different from the generic behaviour of glass forming liquids where local crystalline order is not prominent. For liquids with MRCO, the growth of dynamic and static length scales tracks very closely the MRCO length scale. The growth of relaxation times in these systems are apparently controlled by the growth of the MRCO length scale. On the other hand, for generic glass formers where the growth of MRCO as temperature or density is varied is not prominent, the dynamic length scales grow more rapidly than static length scales and the increase in relaxation times is controlled by the static length scale. Finally we have conclusively shown that the point-to-set correlation method is indeed order agnostic and captures the relevant length scale for all model systems including those with prominent MRCO.
[1] L. Berthier and G. Biroli, "Theoretical perspective on the glass transition and amorphous materials," Rev. Mod. Phys., vol. 83, pp. 587-645, Jun 2011.

[2] S. Karmakar, C. Dasgupta, and S. Sastry, "Growing length scales and their relation to timescales in glassforming liquids," Annu. Rev. Condens. Matter Phys., vol. 5, pp. 255-284, Mar 2014.

[3] S. Karmakar, C. Dasgupta, and S. Sastry, "Length scales in glass-forming liquids and related systems: a review,"
Reports on Progress in Physics, vol. 79, p. 016601, Dec 2015.

[4] M. D. Ediger, "Spatially heterogeneous dynamics in supercooled liquids.," Annual Review of Physical Chemistry, vol. 51, pp. 99-128, Oct 2000.

[5] L. Berthier, G. Biroli, J.-P. C. Bouchaud, L. Masri, D. E. L. ote, D. Ladieu, F. Pierno, and M. Pierno, "Direct experimental evidence of a growing length scale accompanying the glass transition," Science, vol. 310, pp. 1797- 
1800, Jun 2005.

[6] P. G. Wolynes and V. Lubchenko, Structural Glasses and Supercooled Liquids: Theory, Experiment, and Applications. John Wiley \& Sons, 2012.

[7] C. P. Royall and S. R. Williams, "The role of local structure in dynamical arrest," PhysicsReports, vol. 560, pp. 1-75, 2015.

[8] C. P. Royall, S. R. Williams, T. Ohtsuka, and H. Tanaka, "Direct observation of a local structural mechanism for dynamic arrest," Nat Mater, vol. 7, pp. 556-561, Jul 2008.

[9] T. Kawasaki, T. Araki, and H. Tanaka, "Correlation between dynamic heterogeneity and medium-range order in two-dimensional glass-forming liquids," Phys. Rev. Lett., vol. 99, p. 215701, Nov 2007.

[10] H. Tanaka, T. Kawasaki, H. Shintani, and K. Watanabe, "Critical-like behaviour of glass-forming liquids," Nat. Mater., vol. 99, pp. 324-331, Apr 2010.

[11] T. Kawasaki and H. Tanaka, "Structural signature of slow dynamics and dynamic heterogeneity in twodimensional colloidal liquids: glassy structural order," Journal of Physics: Condensed Matter, vol. 23, Apr 2011.

[12] J. Russo and H. Tanaka, "Assessing the role of static length scales behind glassy dynamics in polydisperse hard disks," Proc. Natl. Acad. Sci. USA, vol. 112, pp. 69206924, June 2015.

[13] W. Kob, C. Donati, S. J. Plimpton, P. H. Poole, and S. C. Glotzer, "Dynamical heterogeneities in a supercooled lennard-jones liquid," Phys. Rev. Lett., vol. 79, pp. 2827-2830, Oct 1997.

[14] R. Yamamoto and A. Onuki, "Dynamics of highly supercooled liquids: Heterogeneity, rheology, and diffusion," Phys. Rev. E, vol. 58, pp. 3515-3529, Sep 1998.

[15] L. O. Hedges1, R. L. Jack1, J. P. Garrahan, and D. Chandler, "Dynamic order-disorder in atomistic models of structural glass formers," science, vol. 323, pp. 13091313, Mar 2009.

[16] N. Lacevic, W. F. Star, B. T. Schrder, and C. S. Glotzer, "Spatially heterogeneous dynamics investigated via a time-dependent four-point density correlation function," The Journal of Chemical Physics, vol. 119, pp. 73727387, Jan 2003.

[17] T. Hamanaka and A. Onuki, "Heterogeneous dynamics in polycrystal and glass in a binary mixture with changing size dispersity and composition," Phys. Rev. E, vol. 75, p. 041503, Apr 2007.

[18] K. Binder, S. Sengupta, and P. Nielaba, "The liquidsolid transition of hard discs: first-order transition or kosterlitz-thouless-halperin-nelson-young scenario?," Journal of Physics: Condensed Matter, vol. 14, pp. 23232333, Feb 2002.

[19] G. Biroli, J.-P. Bouchaud, A. Cavagna, T. S. Grigera, and P. Verrocchio, "Thermodynamic signature of growing amorphous order in glass-forming liquids," Nat Phys, vol. 99, pp. 771-775, Oct 2008.

[20] G. M. Hocky, T. E. Markland, and D. R. Reichman, "Growing point-to-set length scale correlates with growing relaxation times in model supercooled liquids," Phys. Rev. Lett., vol. 108, p. 225506, Jun 2012.

[21] L. Berthier and G. Biroli, "Theoretical perspective on the glass transition and amorphous materials," Rev. Mod. Phys., vol. 83, pp. 587-645, Jun 2011.

[22] G. Biroli, J.-P. Bouchaud, K. Miyazaki, and D. R. Reichman, "Inhomogeneous mode-coupling theory and grow- ing dynamic length in supercooled liquids," Phys. Rev. Lett., vol. 97, p. 195701, Nov 2006.

[23] V. Lubchenko and G. P. Wolyne, "Theory of structural glasses and supercooled liquids," Annual Review of Physical Chemistry, vol. 58, pp. 235-266, Oct 2006.

[24] F. Ritort and P. Sollich, "Glassy dynamics of kinetically constrained models," Advances in Physics, vol. 52, p. 219342, 2003.

[25] R. Yamamoto and A. Onuki, "Kinetic heterogeneities in a highly supercooled liquid.," J. Phys. Soc. Jpn, vol. 66, pp. 2545-2548, Sep 1997.

[26] M. M. Hurley and P. Harrowell, "Kinetic structure of a two-dimensional liquid," Phys. Rev. E, vol. 52, pp. 16941698, Aug 1995.

[27] C. Donati, J. F. Douglas, W. Kob, S. J. Plimpton, P. H. Poole, and S. C. Glotzer, "Stringlike cooperative motion in a supercooled liquid," Phys. Rev. Lett., vol. 80, pp. 2338-2341, Mar 1998.

[28] W. Kob and H. C. Andersen, "Testing mode-coupling theory for a supercooled binary lennard-jones mixture i: The van hove correlation function," Phys. Rev. E, vol. 51, pp. 4626-4641, May 1995.

[29] U. R. Pedersen, T. B. Schroder, and J. C. Dyre, "Repulsive reference potential reproducing the dynamics of a liquid with attractions," Phys. Rev. Lett., vol. 105, p. 157801 , Oct 2010.

[30] S. Karmakar, E. Lerner, I. Procaccia, and J. Zylberg, "Statistical physics of elastoplastic steady states in amorphous solids: Finite temperatures and strain rates," Phys. Rev. E, vol. 82, p. 031301, Sep 2010.

[31] S. Karmakar, E. Lerner, and I. Procaccia, "Direct estimate of the static length-scale accompanying the glass transition," Physica A: Statistical Mechanics and its Applications, vol. 391, pp. 1001-1008, Jun 2012.

[32] E. Tamborini1, C. P. Royall, and P. Cicuta, "Correlation between crystalline order and vitrification in colloidal monolayers," Journal of Physics: Condensed Matter, vol. 27, p. 194124, Nov 2010.

[33] S. Karmakar, C. Dasgupta, and S. Sastry, "Analysis of dynamic heterogeneity in a glass former from the spatial correlations of mobility," Phys. Rev. Lett., vol. 105, p. 015701, Jul 2010.

[34] S. Karmakar, C. Dasgupta, and S. Sastry, "Comment on "scaling analysis of dynamic heterogeneity in a supercooled lennard-jones liquid"," Phys. Rev. Lett., vol. 105, p. 019801, Jul 2010.

[35] E. Flenner and G. Szamel, "Dynamic heterogeneity in a glass forming fluid: Susceptibility, structure factor, and correlation length," Phys. Rev. Lett., vol. 105, p. 217801, Nov 2010.

[36] S. Karmakar, C. Dasgupta, and S. Sastry, "Growing length and time scales in glass-forming liquids," Proc. Natl. Acad. Sci. USA, vol. 106, pp. 3675-3679, Jan 2009.

[37] K. Binder, "Finite size scaling analysis of ising model block distribution functions," Zeitschrift fr Physik B Condensed Matter, vol. 43, pp. 119-140, 1981.

[38] F. W. Starr, J. F. Douglas, and S. Sastry, "The relationship of dynamical heterogeneity to the adam-gibbs and random first-order transition theories of glass formation," The Journal of Chemical Physics, vol. 138, Mar 2013.

[39] J.-P. Bouchaud and G. Biroli, "On the adam-gibbskirkpatrick-thirumalai-wolynes scenario for the viscosity increase in glasses," The Journal of Chemical Physics, vol. 121, pp. 7347-7354, July 2004. 
[40] B. Zhang and X. Cheng, "Structures and dynamics of glass-forming colloidal liquids under spherical confinement," Phys. Rev. Lett., vol. 116, p. 098302, Mar 2016.

[41] G. Biroli, S. Karmakar, and I. Procaccia, "Comparison of static length scales characterizing the glass transition," Phys. Rev. Lett., vol. 111, p. 165701, Oct 2013.

[42] S. Yaida, L. Berthier, P. Charbonneau, and G. Tarjus, "Point-to-set lengths, local structure, and glassiness," Phys. Rev. E, vol. 94, p. 032605, Sep 2016. 


\title{
Glass Transition in Supercooled Liquids with Medium Range Crystalline Order - Supplementary Information
}

\author{
Indrajit Tah ${ }^{1}$, Shiladitya Sengupta ${ }^{2}$, Srikanth Sastry ${ }^{3}$, Chandan Dasgupta ${ }^{4}$, and Smarajit Karmakar ${ }^{1}$ \\ 1 Centre for Interdisciplinary Sciences, Tata Institute of Fundamental Research, \\ 21 Brundavan Colony, Narisingi, Hyderabad, 500075, India, \\ 2 Department of Chemical Physics, Weizmann Institute of Science, Israel, \\ 3 Jawaharlal Nehru Centre for Advanced Scientific Research, Bangalore 560064, India, \\ 4 Centre for Condensed Matter Theory, Department of Physics, \\ Indian Institute of Science, Bangalore, 560012, India
}

\section{MODELS AND SIMULATION DETAILS}

We have studied four different model glass forming liquids in two dimensions. The model details are given below:

2dKA: The model glass former we have studied is the Kob-Anderson 80 : 20 [1] Lenard-Jones Binary mixture. This model was first introduced by Kob and Anderson to simulate $N i_{80} P_{20}$. This model has been studied extensively and found to be a very good glass former in three dimensions. In two dimensions this model shows strong growth of medium range crystalline order (MRCO). The interaction potential is given by

$$
V_{\alpha \beta}(r)=4.0 \epsilon_{\alpha \beta}\left[\left(\frac{\sigma_{\alpha \beta}}{r}\right)^{12}-\left(\frac{\sigma_{\alpha \beta}}{r}\right)^{6}\right]
$$

where $\alpha, \beta \in\{A, B\}$ and $\epsilon_{A A}=1.0, \epsilon_{A B}=1.5, \epsilon_{B B}=$ $0.5 ; \sigma_{A A}=1.0, \sigma_{A B}=0.80, \sigma_{B B}=0.88$. The interaction potential was cut off at $2.50 \sigma_{\alpha \beta}$ and the number density is $\rho=1.20$. Length, energy and time scale are measured in units of $\sigma_{A A}, \epsilon_{A A}$ and $\sqrt{\frac{\sigma_{A A}^{2}}{\epsilon_{A A}}}$. For Argon these units corresponds to a length of $3.4 \AA$, an energy of $k_{B}(120 K)$ and time of $3 \times 10^{-13} s$. We have done simulation in the temperature range $T \in\{0.930,2.200\}$.

2dIPL: This model glass forming liquid is the two dimensional version of the Inverse Power Law model studied in [2]. Here the interaction potential is given by

$$
V_{\alpha \beta}(r)=1.945 \epsilon_{\alpha \beta}\left[\left(\frac{\sigma_{\alpha \beta}}{r}\right)^{15.48}\right]
$$

All the parameters and the interaction cut-off are the same as those for the $2 \mathrm{dKA}$ model. The temperature of the system is fixed at $T=0.025$. We have performed simulations in the density range $\rho \in\{0.65,0.75\}$.

2dR10: This is a 50:50 binary mixture [3] with the pair wise interaction potential

$$
V_{\alpha \beta}(r)=\epsilon_{\alpha \beta}\left[\left(\frac{\sigma_{\alpha \beta}}{r}\right)^{10}\right]
$$

Here $\epsilon_{\alpha \beta}=1.0, \sigma_{A A}=1.0, \sigma_{A B}=1.22, \sigma_{B B}=1.40$. The interaction potential is cut-off at $1.38 \sigma_{\alpha \beta}$. The number density $\rho=0.85$ and the temperature range is $T \in\{0.480,1.000\}$.
2dPoly: This is a polydisperse mixture of soft disks with the diameter $\sigma$ of the disks chosen from a Gaussian distribution. The polydispersity $(\Delta)$ of the model is defined as

$$
\Delta=\frac{\sqrt{\left\langle\sigma^{2}\right\rangle-\langle\sigma\rangle^{2}}}{\langle\sigma\rangle}
$$

For our case we fix $\Delta=11 \%$. The particles in this polydisperse model systems interact with the Weeks-ChandleAnderson potential [4]

$$
V_{i j}(r)=4.0 \epsilon_{i j}\left\{\left(\frac{\sigma_{i j}}{r}\right)^{12}-\left(\frac{\sigma_{i j}}{r}\right)^{6}+\frac{1}{4}\right\}
$$

for $r<2^{1 / 6} \sigma_{i j}$, otherwise $V_{i j}(r)=0$, where $\sigma_{i j}=$ $\frac{\left(\sigma_{i}+\sigma_{j}\right)}{2}$.

We study the behavior of the polydisperse system at different temperatures in the range $T \in\{0.450,0.900\}$. While calculating the static length scale using finite size scaling of $\tau_{\alpha}$ (see Sec $7 \mathrm{~B}$ for further details), we fix the packing fraction to be the same for all system sizes. The packing fraction $\phi$ is defined as $\phi=\frac{1}{L^{2}} \sum_{i=1}^{N} \pi\left(\frac{\sigma_{i}}{2}\right)^{2}$ where $L$ is the box size. We fix the packing fraction $\phi=0.76$, the corresponding number density being $\approx 0.95$.

\section{CALCULATION OF $\xi_{6}$}

In this section we describe the calculation of the hexatic order correlation length $\xi_{6}$. We calculate the bond orientational order for each particle $[5,6]$ as

$$
\psi_{6}^{j}=\frac{1}{n_{j}} \sum_{k=1}^{n_{j}} \exp \left(i 6 \theta_{j k}\right) \text {. }
$$

Here $\psi_{6}^{j}$ is the hexatic order parameter for the $j^{t h}$ particle, $n_{j}$ is the number of nearest neighbours of particle $j$, and $\theta_{j k}$ is the angle made by the position vectors of two particles $j$ and $k$ with a reference axis. Here we have taken the cutoff nearest neighbour distance to be the distance of the first minimum of the pair distribution function $g(r)$.

The snapshots in Fig. 1, Fig. 2 and Fig. 3 show the growth of hexatic order from high temperature to low 

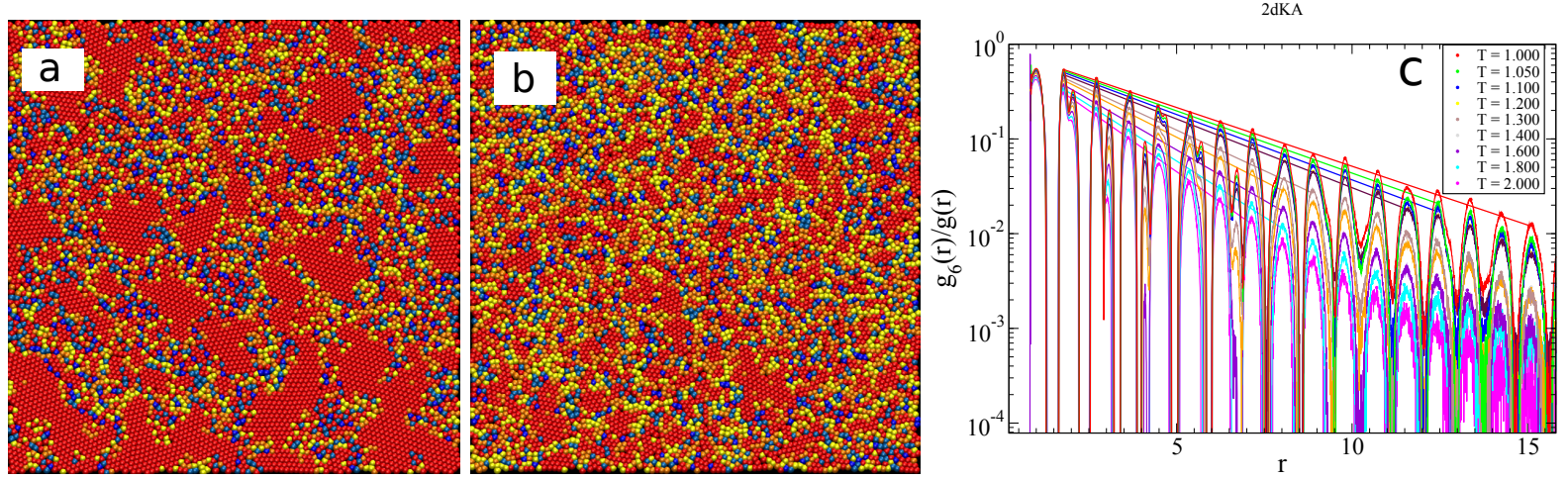

FIG. 1. Snapshots of configurations for the $2 \mathrm{dKA}$ model (a) at a low temperature, $\mathrm{T}=1.000$ and (b) at a high temperature, $\mathrm{T}$ $=2.000$. The particles are colored according the hexatic order parameter. Red corresponds to higher values of $\left|\psi_{6}^{i}\right|$. The size of the correlation region increases with decreasing temperature. (c) Decay of the hexatic order correlation function $g_{6}(r) / g(r)$ for $2 \mathrm{dKA}$ systems. The solid straight lines are obtained by fitting the peaks of the correlation function to the $2 \mathrm{D}$ version of the Ornstein-Zernike (OZ) function.
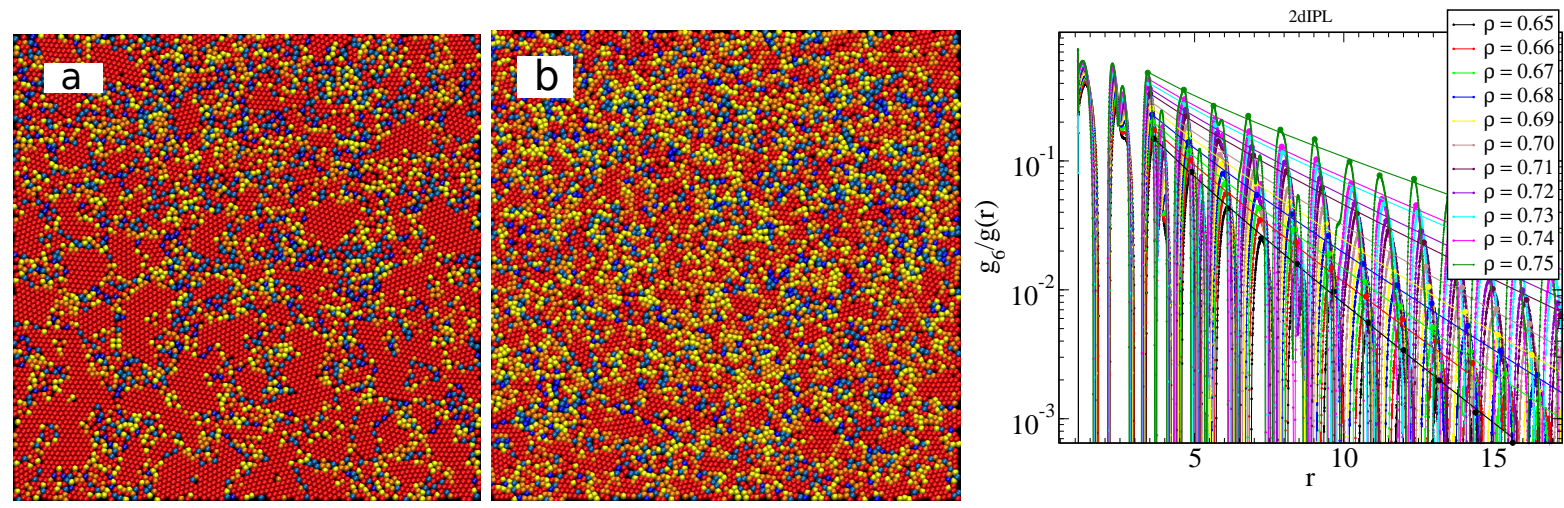

FIG. 2. Snapshots of configurations for the 2dIPL model (a) at high density, $\rho=0.74$ and (b) at low density, $\rho=0.67$. The particles are colored according the hexatic order parameter. Red corresponds to higher values of $\left|\psi_{6}^{i}\right|$. The size of the correlation region increases with increasing density. (c) Decay of the hexatic order correlation function $g_{6}(r) / g(r)$ for $2 \mathrm{dIPL}$ systems. The solid straight lines are obtained by fitting the peaks of the correlation function to the $2 \mathrm{D}$ version of the Ornstein-Zernike (OZ) function.
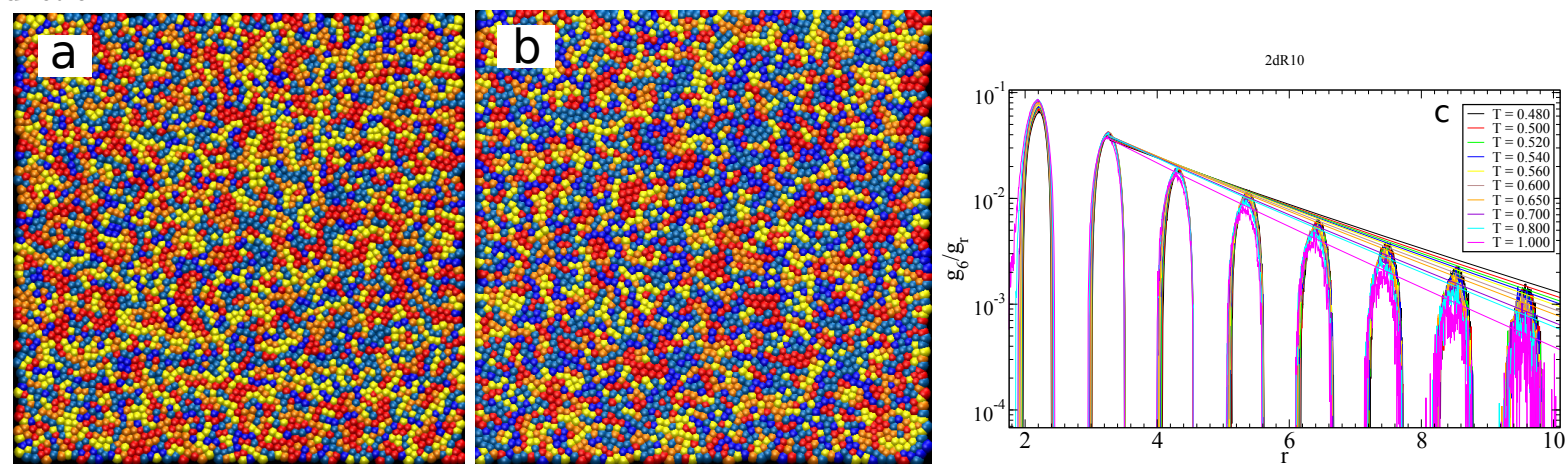

FIG. 3. Snapshots of configurations for the $2 \mathrm{dR} 10$ model (a) at a low temperature, $\mathrm{T}=0.500$ and (b) at a low temperature, $\mathrm{T}=1.000$. The particles are colored according the hexatic order parameter. Red corresponds to higher values of $\left|\psi_{6}^{i}\right|$. Here, there is no growth of local crystalline order with decreasing temperature. (c) Decay of the hexatic order correlation function $g_{6}(r) / g(r)$ for $2 \mathrm{dR} 10$ systems. The solid straight lines are obtained by fitting the peaks of the correlation function to the $2 \mathrm{D}$ version of the Ornstein-Zernike (OZ) function. 
temperature (2dKA and 2dR10 models) and low density to high density (2dIPL model). For the 2dR10 model it is clearly seen from Fig. 3 that there is no prominent growth of hexatic order at low temperatures and for the other two models (2dKA and 2dIPL) there is prominent growth of hexatic order at low temperatures and high densities. In these figures we use the same colour scheme as described in the main text.

By defination $\left|\psi_{6}^{j}\right|=1$ for a perfect triangular lattice and $\left|\psi_{6}^{j}\right|<1$ implies that the structure deviates from the perfect triangular lattice configuration. We calculate the spatial correlation of local hexatic order, $g_{6}(r)[7,8]$ as:

$$
g_{6}(r)=\frac{L^{2}}{2 \pi r \Delta r N(N-1)} \sum_{j \neq k} \delta\left(r-\left|r_{j k}\right|\right) \psi_{6}^{j} \psi_{6}^{k *}
$$

The hexatic correlation length $\xi_{6}$ is extracted by fitting the peak values of the normalized hexatic correlation function, $g_{6}(r) / g(r)$. We have also used the two dimensional version of the Ornstein-Zernike (OZ) function to fit the peaks of the correlation function. In the right panels of Figs.1, 2 and 3 we show the hexatic order correlation function for the three model systems.

\section{CALCULATION OF DYNAMICAL LENGTH SCALES}

Binder Cummulant: We calculate the dynamical correlation length $\xi_{d}$ in various ways to assess the robustness of our results. We extract the dynamical length scale $\xi_{d}[9,10]$ from finite size scaling of the peak height of the four-point dynamic susceptibility $\chi_{4}(t)$. Four-point dynamic correlations (defined below) can be thought of as the correlation between two relaxation processes at two spatial points separated by some distance and the dynamical susceptibility $\chi_{4}(t)$ is the integrated effect of these correlations over the whole volume. The relaxation processes are characterized by the two point correlation function $Q(t)$, which gives the amount of overlap between two configurations which are separated by time t,

$$
Q(t)=\sum_{i=1}^{N} w\left(\left|r_{i}(0)-r_{i}(t)\right|\right)
$$

where $w(r)=1$ when $r \leq a$ and 0 otherwise. The parameter $a$ is chosen to eliminate from consideration the decorrelation that might arise due to the vibrational motion of particles inside the cages formed by other, neighboring, particles. The particular choice of $a$ is not very important, and in our studies we choose $a=0.3$ which corresponds to the plateau value of the mean square displacement.

The four point susceptibility, $\chi_{4}(t)$, [11] is defined as

$$
\left.\chi_{4}(t)=\frac{1}{N}\left[\left\langle Q^{2}(t)\right\rangle\right]-\langle Q(t)\rangle^{2}\right]
$$
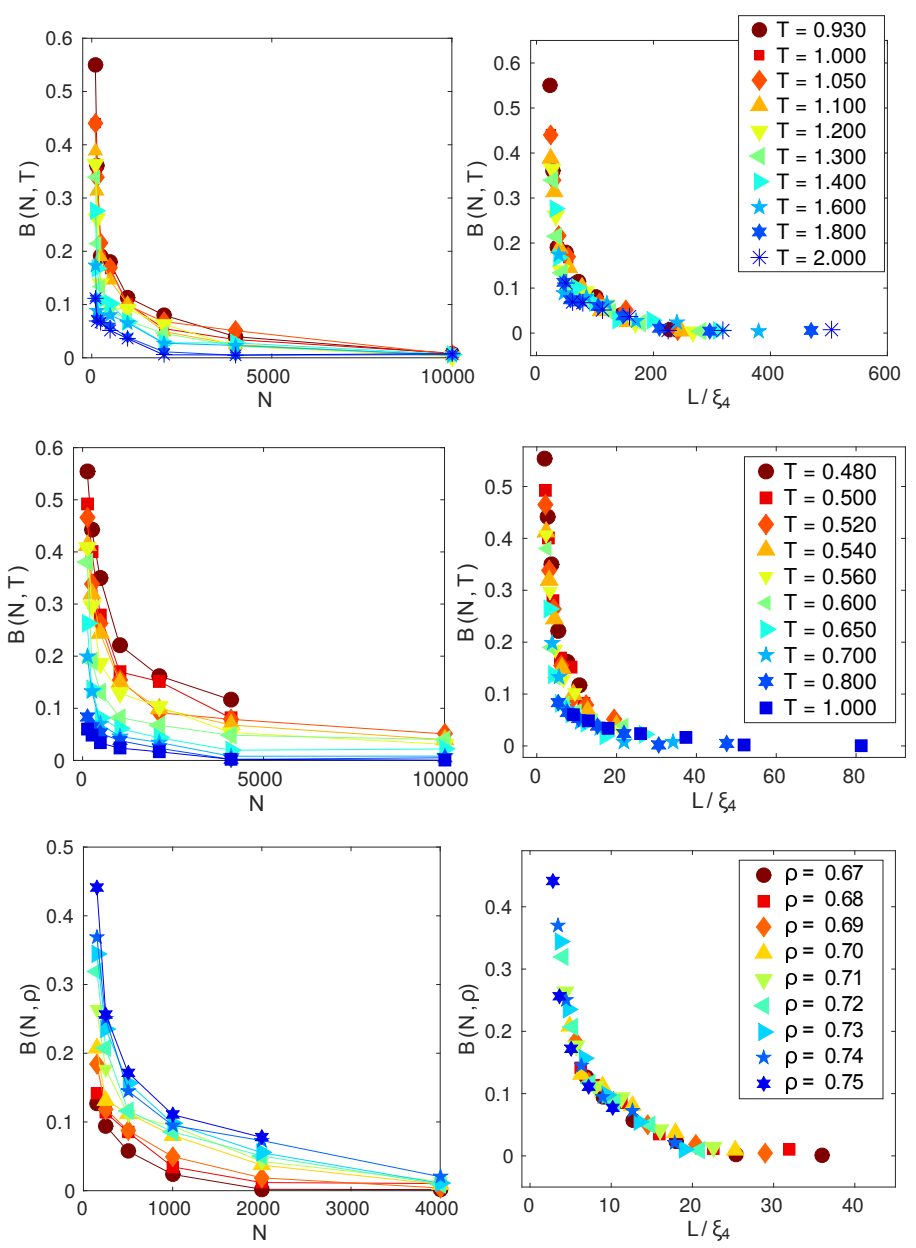

FIG. 4. Left panel shows the Binder cumulant B(N,T) and $B(N, \rho)$ as a function of system size for the three systems. The top panel is for $2 \mathrm{dKA}$, the middle one is for $2 \mathrm{dR} 10$ and the bottom one is for $2 \mathrm{dIPL}$ model systems. The right panel shows the data collapse for $\mathrm{B}(\mathrm{N}, \mathrm{T})$ and $B(N, \rho)$ plotted as a function of $L / \xi$. The correlation length $\xi$ is an unknown quantity which is determined by requiring data collapse for the values of the Binder cumulant at different temperature or density and system size.

Finite size scaling (FSS) is done for the system size dependence of the peak value of $\chi_{4}(t), \chi_{4}^{P}$ for each temperature using the following scaling ansatz

$$
\chi_{4}^{P}(N, T)=\chi_{4}^{P}(\infty, T) \mathcal{F}\left(\frac{N}{\xi_{d}^{d}}\right),
$$

where $\chi_{4}^{P}(\infty, T)$ is the asymptotic value of $\chi_{4}^{P}$ in the infinite system size limit. In this scaling analysis both $\chi_{4}^{P}(\infty, T)$ and the length scale $\xi_{d}$ are treated as parameters to be determined by demanding data collapse. $\mathcal{F}(x)$ is the unknown scaling function.

A better method to estimate this dynamical length scale $\xi_{d}$ is from the Binder cumulant [12] which is defined in terms of the fourth and second moments of the distribution of $Q(\tau)$ where $\tau$ is the time associate with 
peak height of $\chi_{4} . \quad \tau$ is proportional to the structural relaxation time $\tau_{\alpha}$ with a proportionality constant very close to 1 . The binder cumulant is defined as

$$
B(N, T)=1-\frac{\left\langle[Q(\tau)-\langle Q(\tau)\rangle]^{4}\right\rangle}{3\left\langle[Q(\tau)-\langle Q(\tau)\rangle]^{2}\right\rangle^{2}} .
$$

By its definition, $B(N, T)=0$ for a Gaussian distribution of $P(Q(\tau))$ and it is a scaling function of only $N / \xi_{d}^{d}$ without any pre-factor:

$$
B(L, T)=\mathcal{G}\left(\frac{L}{\xi_{d}}\right)
$$

where $L=(N / \rho)^{1 / d}$ is the linear size of the system, $\xi_{d}$ is the correlation length and $\mathcal{G}(x)$ is the scaling function. In Fig. 4 (left panels) we show the system size dependence of the Binder Cumulant at different temperatures and densities for the 2dKA, 2dIPL and 2dR10 models. Right panels of the same figures show the data collapse achieved by estimate values of the dynamical length scales $\xi_{d}$. The observed good data collapse confirms the reliability of the extracted length scales.

Four-point Structure Factor: The four point structure factor [13] is defined as

$$
S_{4}(q, \tau)=N\left[\langle\tilde{Q}(q, \tau) \tilde{Q}(-q, \tau)\rangle-|\langle\tilde{Q}(q, \tau)\rangle|^{2}\right] .
$$

Here, the spatial Fourier decomposition of the local mobility is defined as

$$
\tilde{Q}(q, t)=\frac{1}{N} \sum_{i=1}^{N} \exp \left(i \mathbf{q} \cdot \mathbf{r}_{i}(o)\right) w\left(\left|\vec{r}_{i}(t)-\vec{r}_{i}(0)\right|\right) .
$$

We plot the $S_{4}(q, \tau)$ as a function $q$ in Fig. 5. We use the Ornstein-Zernike (OZ) form for the $q$ dependence of $S_{4}(q, \tau)$ :

$$
S_{4}(q, \tau)=\frac{S_{4}(q=0, \tau)}{1+\left[q \xi_{4}\right]^{2}}
$$

where $S_{4}(q=0, \tau)$ and $\xi_{4}$ are treated as fitting parameters. We find a good fit in the range $q \in[0.097,0.486]$ for the $2 \mathrm{dKA}$ model system, $q \in[0.076,0.384]$ for the $2 \mathrm{dIPL}$ system and $q \in[0.058,0.492]$ for the $2 \mathrm{dR} 10$ system. The system size used for this analysis is $N=10000$. One finds the fits to the $\mathrm{OZ}$ equation to be very good and the obtained length-scales are in good agreement with the ones obtained using FSS of $\chi_{4}^{P}$ and the Binder cummulant.

Four-point Structure Factor from Bond-breakage Correlation: The dynamical length scale $\xi_{d}$ is also calculated (for the 2dKA model) from the susceptibility of a bond breakage correlation function following the procedure of Ref.[14, 15]. It was pointed out in [14] that in two dimensions, due to long wave length density fluctuations (consequence of Mermin-Wagner Theorem), $\chi_{4}$ and the corresponding structure factor $S_{4}(q, \tau)$ show a strong contribution from phonon like modes. This masks the
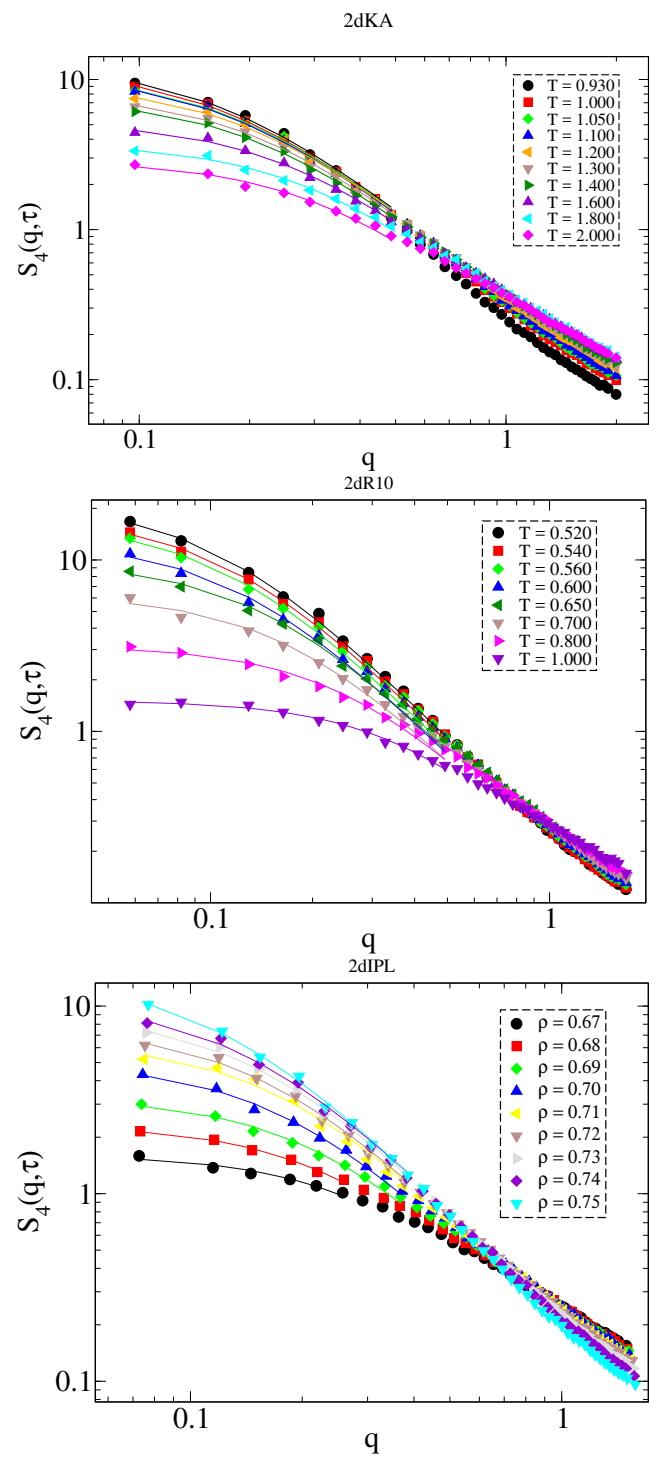

FIG. 5. $S_{4}(q, \tau)$ for $2 \mathrm{dKA}$ (for different temperature), $2 \mathrm{dR} 10$ (for different temperature) and 2dIPL (for different density). The solid lines are fits to the Ornstein-Zernike function.

contribution of the growth of the dynamic length scale and extracting the dynamic length scale from $S_{4}\left(q, \tau_{4}\right)$ becomes erroneous. So to check the reliability of the extracted dynamic heterogeneity length scale from different methods, we have also calculated the same using the four-point structure factor from the bond-breakage correlation function.

The bond-breakage correlation function is defined as follows. Initially we consider a pair of atom $i$ and $j$ to be bonded if

$$
r_{i j}\left(t_{0}\right)=\left|\mathbf{r}_{i}\left(t_{0}\right)-\mathbf{r}_{j}\left(t_{0}\right)\right| \leq 1.0 \sigma_{\alpha \beta} .
$$

where $i$ and $j$ belong to the species $\alpha$ and $\beta$ with $\sigma_{\alpha \beta}=$ $\frac{1}{2}\left(\sigma_{\alpha}+\sigma_{\beta}\right)$. After some elapsed time we calculate the surviving bonds which satisfy the criterion $r_{i j}\left(t_{0}+t\right) \leq$ $1.35 \sigma_{\alpha \beta}$. The bond breakage correlation is defined as 
the number of surviving bonds normalized by the total number of bond at the initial time. From this correlation function, we extract the bond breakage time, $t_{B}$, as the time at which the correlation function becomes $1 / e$ of its initial value.

We next calculate the structure factor $S_{B}\left(q, t_{B}\right)$ which is defined as

$$
S_{B}\left(q, t_{B}\right)=\frac{1}{N_{b}}\left\langle\left|\sum \exp \left(i \mathbf{q} \cdot \mathbf{R}_{i j}\right)\right|^{2}\right\rangle_{a}
$$

where the summation runs over broken pairs, $N_{b}$ is the number of broken bonds and $\mathbf{R}_{i j}=\frac{1}{2}\left(\mathbf{r}_{i}\left(t_{0}\right)+\mathbf{r}_{j}\left(t_{0}\right)\right)$ is the center position of the broken pair at the initial time $t_{0} . \quad\langle. .\rangle_{a}$ is the angular average over the direction of the wave vector $\mathbf{q}=\frac{2 \pi}{L}\left(n_{x}, n_{y}\right)$ where $n_{x}, n_{y}=$ $\pm 1, \pm 2, \pm 3, \ldots$ Then we plot the $S_{B}\left(q, t_{B}\right)$ as a function $\mathrm{q}$ in Fig. 6. We use the same Ornstein-Zernike (OZ) form to fit the function.

$$
S_{B}\left(q, t_{B}\right)=\frac{S_{B}\left(q=0, t_{B}\right)}{1+\left[q \xi_{B}\right]^{2}}
$$

$S_{B}\left(q, t_{B}\right)$ and the $\xi_{B}$ obtained from the fits exhibit no significant size dependence. The obtained length scale $\xi_{B}$ is also in good agreement with the dynamical length scales obtained from the Binder cumulant.

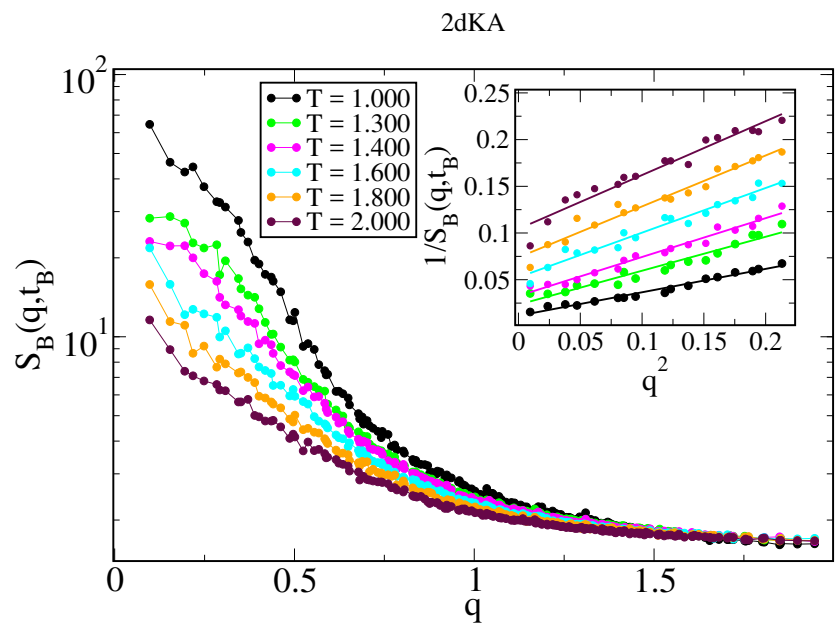

FIG. 6. $S_{B}(q)$, the bond-beakage structure factor, for the 2dKA model. The inset shows $1 / S_{B}\left(q, t_{B}\right)$ vs $q^{2}$ from which $\xi_{B}$ is determined.

\section{STATIC LENGTH SCALE FROM FINITE SIZE SCALING OF $\tau_{\alpha}$}

We calculate the static length scale from finite size scaling of the $\alpha$ relaxation time, $\tau_{\alpha}$ [16]. The relaxation time $\tau_{\alpha}$ is defined from $Q\left(\tau_{\alpha}\right)=1 / e$. The $\alpha$ relaxation time shows strong system size dependence, especially at low temperatures. It decreases monotonically with increasing system size and reaches an asymptotic value at large system sizes. The system sizes dependence of the $\alpha$ relaxation time becomes more prominent at low temperatures or at high densities. We use the following scaling form to obtain the static length scale:

$$
\tau_{\alpha}(N, T)=\tau_{\alpha}(\infty, T) \mathcal{G}\left(\frac{N}{\xi_{s}^{d}(T)}\right),
$$

where the form of $\mathcal{G}(x)$ is unknown and one does not need the details of this scaling function to obtain the static length scale $\xi_{s}$. In the left panels of Fig. 7, we plot $\tau_{\alpha}(N, T)$ scaled by $\tau_{\alpha}(\infty, T)$ for the $2 \mathrm{dKA}$ and $2 \mathrm{dR} 10$ models and $\tau_{\alpha}(N, \rho)$ scaled by $\tau(\infty, \rho)$ for the 2dIPL model as a function of system size, $N$. To estimate the value of $\tau_{\alpha}(\infty, T)$ or $\tau_{\alpha}(\infty, \rho)$ we have used the following functional form $f(x)=a+b / x$. The right panels show the data collapse for the three model systems, which is observed to be good. Reasonably good collapse of the data gives confidence in the reliability of the extracted static length scale $\xi_{s}$.
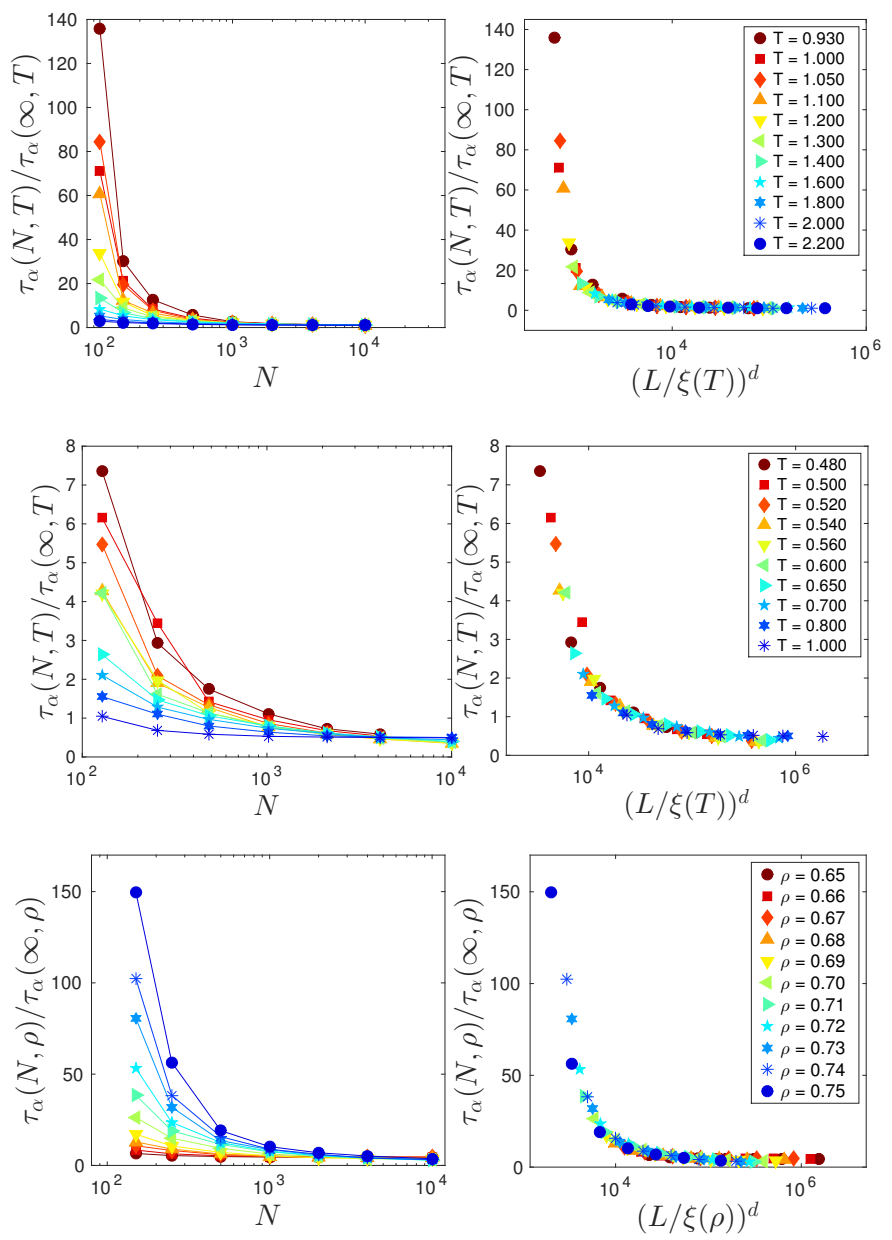

FIG. 7. Top panel shows the finite system size dependence of the $\alpha$ relaxation time for the $2 \mathrm{dKA}$ model and data collapse to obtain the static length scale. The middle one shows the same analysis for the $2 \mathrm{dR} 10$ model system and the bottom one for the 2dIPL system. 


\section{STATIC LENGTH SCALE FROM FINITE SIZE SCALING OF THE MINIMUM EIGENVALUE OF THE HESSIAN MATRIX}
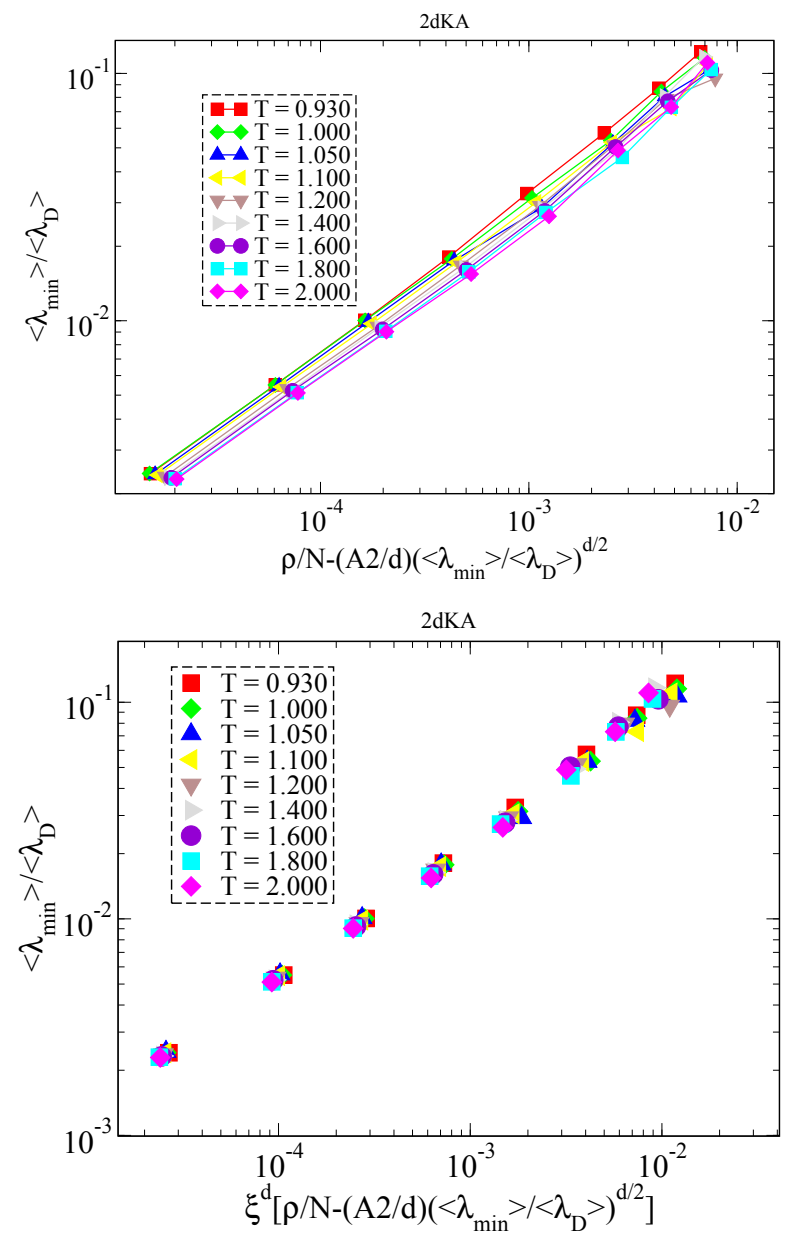

FIG. 8. Scaling of the minimal eigenvalue calculated for the 2dKA model.

Yet another method used to obtain the static length scale is the finite size scaling of the minimum eigenvalue, $\lambda_{\min }$ of the Hessian matrix [3] evaluated at the minima of the potential energy (Inherent Structure or IS) explored by the system at a given temperature or density. It is argued in [3], that $\lambda_{\min }$ will have a cross-over in the system size dependence with increasing system sizes and the cross over length scale is the static length scale. This method is useful for low temperature or at high density. At high temperature or low density, due to an-harmonic effects, the scaling arguments do not hold and the obtained length scale becomes increasingly less reliable. To obtain the static length scale we have used the following scaling form as suggested in [3]:

$$
\frac{\left\langle\lambda_{\min }(T)\right\rangle}{\left\langle\lambda_{D}(T)\right\rangle}=\mathcal{F}\left[\xi_{s}^{d}(T)\left(\frac{1}{V}-\frac{A 2}{d}\left(\frac{\left\langle\lambda_{\min }(T)\right\rangle}{\left\langle\lambda_{D}(T)\right\rangle}\right)^{d / 2}\right)\right],
$$

where $\mathcal{F}$ is an unknown scaling function and $\lambda_{D} \approx$ $\mu \rho^{(2 /(d-1))}$ where $\rho$ is the Debye frequency and $\mu$ is the shear modulus. We obtain the length scale $\xi_{s}$ by demanding that all data should collapse onto a master curve for different system sizes and temperature (or density) by correctly choosing $\xi_{s}(T)$. In the top panel of Fig. 8, we plot the data according to the above scaling ansatz. In the bottom panel, we show the collapsed data in a master curve to extract the correlation length scale. Similar data for the $2 \mathrm{dR} 10$ model are taken from Ref. [3].

\section{POINT TO SET LENGTH SCALE:}

To measure the length scale $\xi_{p t s}$, we follow the methods elaborated in Refs.[17, 18]. First, we equilibrate a system of $N$ particles and then cavities are generated by freezing the particles outside a sphere of radius $R$. We study how the thermodynamics of the particles inside the sphere of radius $R$ is affected by the amorphous boundary condition generated by the pinned particles. While choosing the cavities we demand that the density or packing fraction of the set of particles inside the cavity should be equal to the bulk density or packing fraction within a tolerance of $2 \%$.

We then partition the central region of the cavity into $M$ cubic boxes of side $l$ to calculate the static overlap defined below. The size of the boxes is such that probability of finding more than one particle in a single box is negligibly small. The static overlap is defined as

$$
q_{c}(R)=\lim _{t \rightarrow \infty} \frac{1}{M l^{2} \rho} \sum_{i=1}^{M}\left\langle n_{i}(0) n_{i}(t)\right\rangle .
$$

Here $\langle.$.$\rangle implies both thermal average and ensemble av-$ erage. The maximum overlap so defined of two configurations is 1 and that of two uncorrelated configurations is $q_{0}=\rho l^{2}$. To extract the PTS length scale we choose $M=6 \times 6$ boxes of side $l=0.36$. We use the particle swap annealing (PSA) method [18, 19] with molecular dynamics simulation to extract the overlap correlation function for different cavity sizes. The length scale $\xi_{p t s}$ is obtained by fitting the overlap function with a compressed exponential form as given below

$$
\tilde{q}(R)=q_{c}(R)-q_{0}=A \exp \left[-\left(\frac{R-a}{\xi_{p t s}}\right)^{\eta}\right],
$$

where $A$ is a fixed number for all temperatures following [18]. In our case, we find that $A=0.55$ fits the data well for the 2dKA, 2dR10 and 2dIPL model systems.

\section{POLYDISPERSE SYSTEM: 2DPOLY}

In this section some of the details of our results for the polydisperse system (2dPoly) are given. 

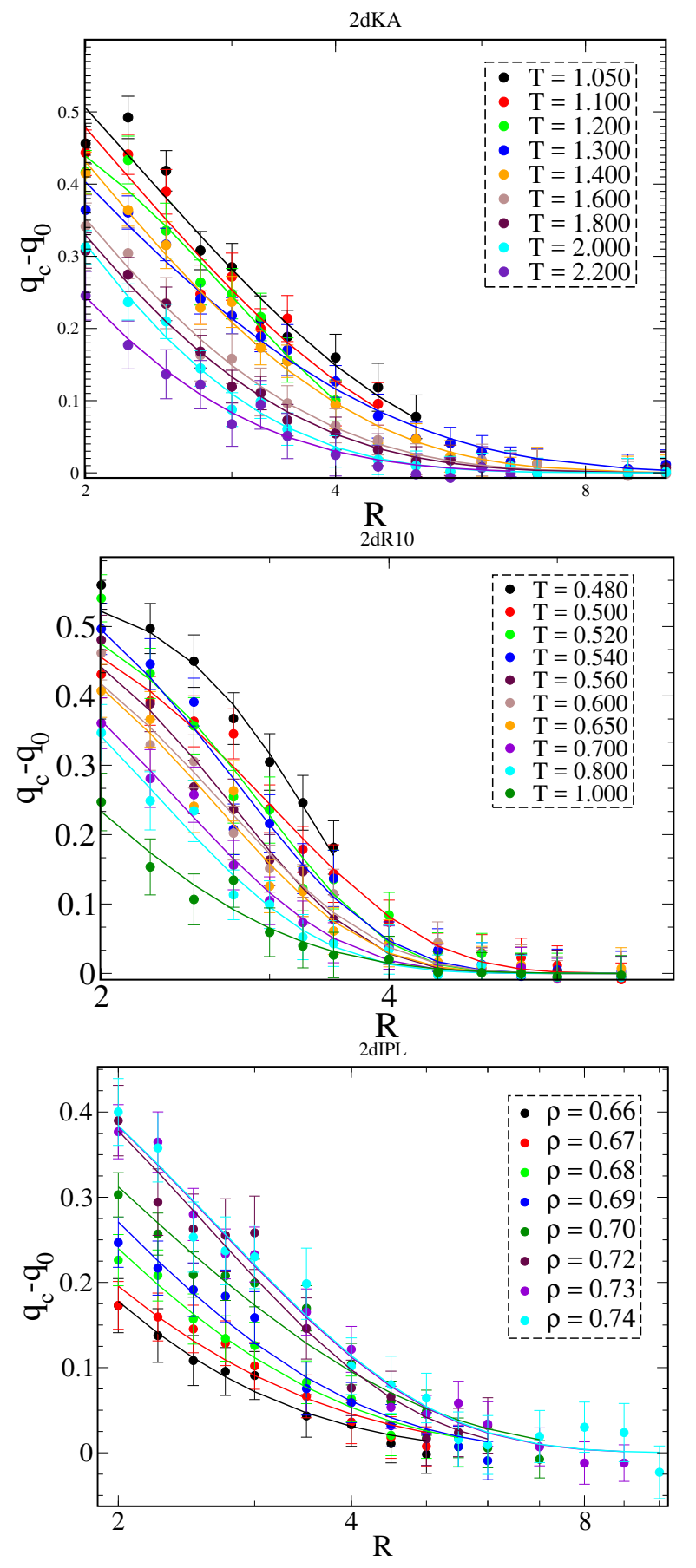

FIG. 9. Overlap as a function of cavity size $\mathrm{R}$ for different temperature and density for the three model systems. Lines are compressed exponential fits of the data with the form discussed in text.

\section{A. Hexatic and Dynamic correlation length scale:}

For the calculation of the hexatic correlation length $\xi_{6}$ we follow the same procedure as described in Sec.2. In the top panel of Fig. 10 we show the hexatic correlation function normalized by $g(r)$ for the 2 dPoly model. The lines are fits of the peak values of $g_{6}(r) / g(r)$ to the $\mathrm{OZ}$ form, which we employ to extract the hexatic correlation length, $\xi_{6}$. In the bottom panel of Fig.10 we plot $S_{4}(q, \tau)$ as a function of $q$. We find a good fit to the $\mathrm{OZ}$ form in the region of $q \in[0.0614,0.3935]$ for this model. From this we obtain the dynamical length scale $\xi_{4}$.
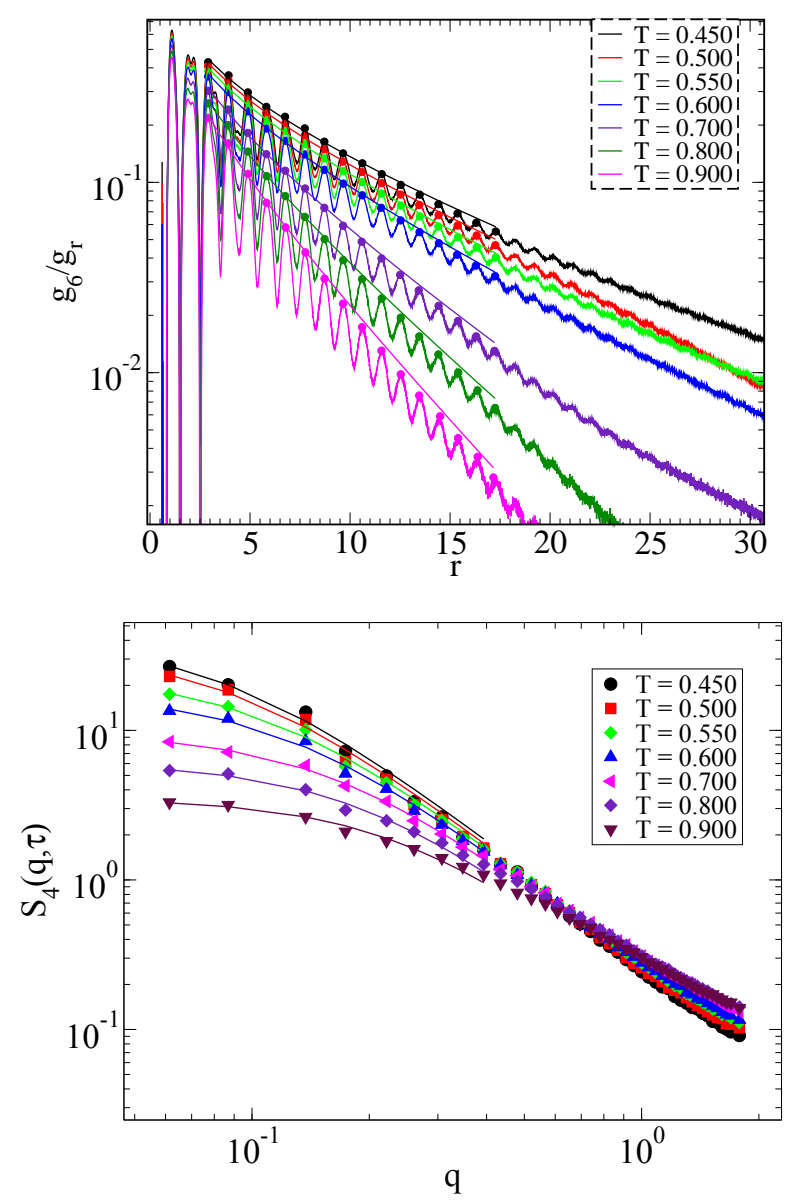

FIG. 10. Top panel: Decay of the hexatic order correlation function $g_{6}(r) / g(r)$ for the 2 d polydisperse system. Bottom panel: $S_{4}(q, \tau)$ for the 2 d polydisperse system for different temperatures. The solid lines are fits with the OrnsteinZernike function.

\section{B. Static Length-scales}

Finite size scaling of $\tau_{\alpha}$ : We have calculated the static length scale from finite size scaling of the $\alpha$ relaxation time $\tau_{\alpha}$ following the procedure of Sec.4. In Fig. 11 we show the finite size scaling of $\tau_{\alpha}$.

Point-to-set length scale: For calculating the pointto-set length scale we follow the procedure described in Sec.6.

The point-to-set analysis for the polydisperse system is demanding. For this model system it is very difficult to obtain the density or the packing fraction to be close to 

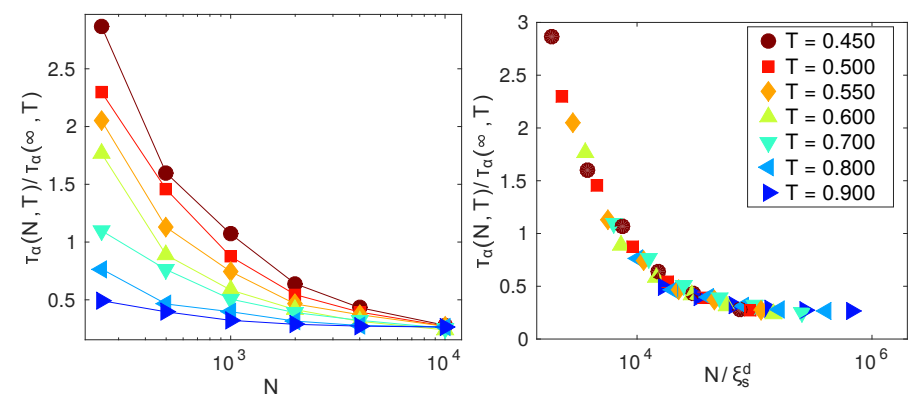

FIG. 11. System size dependence of the $\alpha$ relaxation time for the $2 \mathrm{~d}$ polydisperse model system (left panel) and data collapse to obtain the static length scale (right panel).
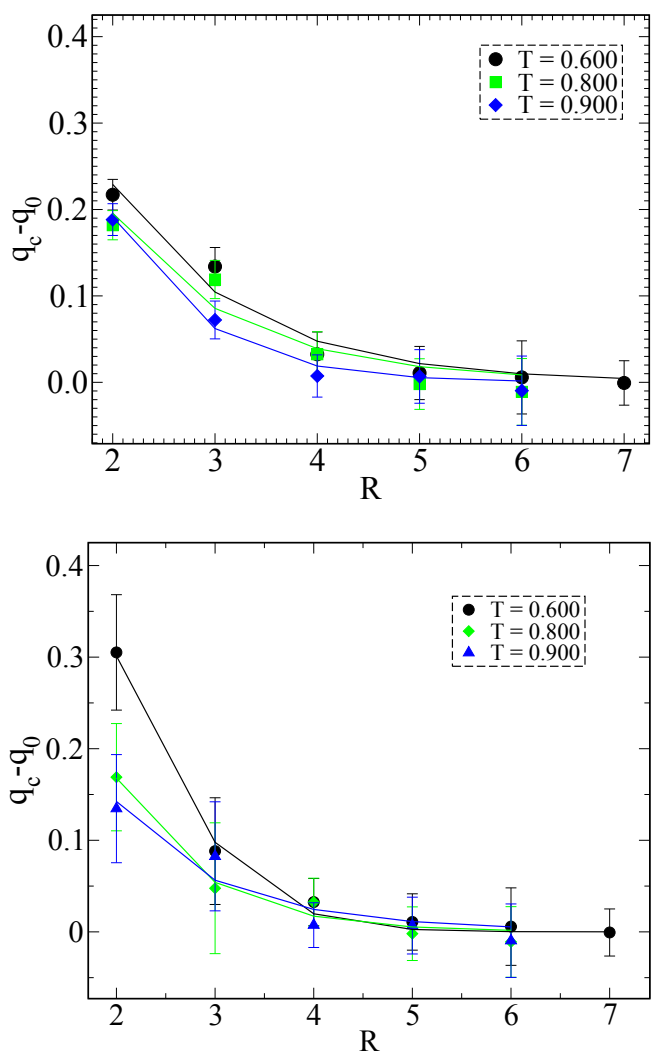

FIG. 12. Top panel shows the overlap as a function of cavity radius $\mathrm{R}$ for different temperatures, averaged over all 250 cavities. Bottom panel shows the results obtained by considering cavities whose packing fraction is similar to the bulk packing fraction. the bulk value due to large fluctuations of particle sizes in these cavities. This is particularly severe for small cavities and small cavities are the ones for which one expects to have large values of the static overlap in the studied temperature range. Another important feature is the polydispersity inside the cavity, which also has to be constrained to be close to the bulk polydispersity. Obtaining small cavities with packing fraction and polydispersity close to the bulk values requires a large sampling of statistically independent cavities. As described in the main text, not restricting the cavity packing fraction and polydispersity to be close to the bulk values results in point-to-set length scales $\xi_{p t s}$ that show very little variation with temperature.

This result is very similar to the results obtained in Ref.[20]. If we choose only those cavities for which the packing fraction and polydispersity are close to the bulk values, the obtained PTS length scale $\xi_{p t s}$ exhibits stronger temperature dependence which matches the temperature dependence of other static length scales obtained by the methods mentioned above. We have averaged over 250 realizations (for small cavities) in simulations of $N=10000$ particles. When we choose only those cavities for which the packing fraction is close to the bulk value, the number of cavities used in the averaging reduces to approximately 32 , which results in greater uncertainty in the values of the overlap. In Fig.12 we show data for the estimates of the PTS length scale. In the top panel we show the results obtained by taking all cavities irrespective of the packing fraction and the observed correlation seems not to grow much. In the bottom panel we show the data for the case where only those cavities are used in the analysis for which the packing fraction is close to the bulk value. The correlations indeed increase substantially in this case. In this case we have chosen $M=4 \times 4$ boxes of linear size $l=0.35$. We find that $\mathrm{A}=0.50$ fits the data better for this model system.
[1] W. Kob and H. C. Andersen, Phys. Rev. E 52, 4134 (1995).

[2] U. R. Pedersen, T. B. Schrøder, and J. C. Dyre, Phys. Rev. Lett. 105, 157801 (2010).

[3] S. Karmakar, E. Lerner, and I. Procaccia, Physica A: Statistical Mechanics and its Applications 391, 1001 (2012).
[4] D. J. Weeks, D. Chandler, and C. H. Andersen, The Journal of Chemical Physics 54, 5237 (1971).

[5] T. Hamanaka and A. Onuki, Phys. Rev. E 74, 011506 (2006).

[6] K. Binder, S. Sengupta, and P. Nielaba, Journal of Physics: Condensed Matter 14, 2323 (2002).

[7] T. Kawasaki, T. Araki, and H. Tanaka, Phys. Rev. Lett. 
99, 215701 (2007).

[8] T. Kawasaki and H. Tanaka, Journal of Physics: Condensed Matter 23 (2011), 10.1088/0953$8984 / 23 / 19 / 194121$.

[9] S. Karmakar, C. Dasgupta, and S. Sastry, Annual Review of Condensed Matter Physics 5, 255 (2014).

[10] S. Karmakar, C. Dasgupta, and S. Sastry, Proc. Natl. Acad. Sci. USA 106, 3675 (2009).

[11] C. Dasgupta, V. A. Indrani, S. Ramaswamy, and K. M. Phani, Europhys. Lett. 15, 307 (1991).

[12] K. Binder, Zeitschrift fr Physik B Condensed Matter 43, 119 (1981).

[13] N. Lacevic, W. F. Star, B. T. Schrder, and C. S. Glotzer, The Journal of Chemical Physics 119, 7372 (2003).
[14] H. Shiba, Y. Yamada, T. Kawasaki, and K. Kim, Phys. Rev. Lett. 117, 245701 (2016).

[15] R. Yamamoto and A. Onuki, J. Phys. Soc. Jpn. 66, 2545 (1997).

[16] S. Karmakar, C. Dasgupta, and S. Sastry, Reports on Progress in Physics 79, 016601 (2015).

[17] G. Biroli, J.-P. Bouchaud, A. Cavagna, T. S. Grigera, and P. Verrocchio, Nat Phys 99, 771 (2008).

[18] G. M. Hocky, T. E. Markland, and D. R. Reichman, Phys. Rev. Lett. 108, 225506 (2012).

[19] T. S. Grigera and G. Parisi, Phys. Rev. E 63, 045102 (2001).

[20] J. Russo and H. Tanaka, Proc. Natl. Acad. Sci. USA 112, 6920 (2015). 